\title{
Measuring Hydrometeors Using a Precipitation Microphysical Characteristics Sensor: Sampling Effect of Different Bin Sizes on Drop Size Distribution Parameters
}

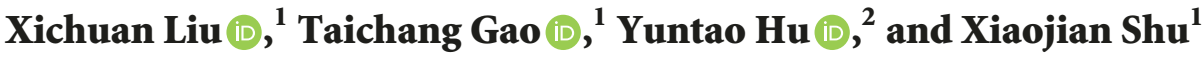 \\ ${ }^{1}$ College of Meteorology and Oceanography, National University of Defense Technology, Nanjing, China \\ ${ }^{2}$ Xichang Satellite Launch Center of China, Xichang 615000, China \\ Correspondence should be addressed to Xichuan Liu; liuxc85@gmail.com
}

Received 26 October 2017; Revised 7 January 2018; Accepted 21 January 2018; Published 26 February 2018

Academic Editor: Youcun Qi

Copyright (c) 2018 Xichuan Liu et al. This is an open access article distributed under the Creative Commons Attribution License, which permits unrestricted use, distribution, and reproduction in any medium, provided the original work is properly cited.

In order to improve the measurement of precipitation microphysical characteristics sensor (PMCS), the sampling process of raindrops by PMCS based on a particle-by-particle Monte-Carlo model was simulated to discuss the effect of different bin sizes on DSD measurement, and the optimum sampling bin sizes for PMCS were proposed based on the simulation results. The simulation results of five sampling schemes of bin sizes in four rain-rate categories show that the raw capture DSD has a significant fluctuation variation influenced by the capture probability, whereas the appropriate sampling bin size and width can reduce the impact of variation of raindrop number on DSD shape. A field measurement of a PMCS, an OTT PARSIVEL disdrometer, and a tipping bucket rain Gauge shows that the rain-rate and rainfall accumulations have good consistencies between PMCS, OTT, and Gauge; the DSD obtained by PMCS and OTT has a good agreement; the probability of $N_{0}, \mu$, and $\Lambda$ shows that there is a good agreement between the Gamma parameters of PMCS and OTT; the fitted $\mu-\Lambda$ and $Z-R$ relationship measured by PMCS is close to that measured by OTT, which validates the performance of PMCS on rain-rate, rainfall accumulation, and DSD related parameters.

\section{Introduction}

Measurement of hydrometeors microphysical characteristics, such as size, shape, fall velocity, and their spatial distribution, is of high interest in fields of precipitation physics, numerical weather prediction models, ground validation of satellite remote sensing, electromagnetic wave propagation, and other climatological and hydrological applications [1-3]. There are hundreds of papers written describing and discussing the parameterization of drop size distribution (DSD) in space and in time [4].

There are many instruments available to measure the drop size distribution of precipitation, such as the PARSIVEL [5], 2DVD [6], and MASC [7]; these instruments have been widely used and extensively researched. Many comparative observations from above instruments have found that there are different discrepancies between different instruments [813], even in the exactly same rainfall conditions, which makes it difficult to understand the microphysical mechanisms of precipitation and relevant applications. This can be attributed to two factors: sampling effect and instrument parameters; at present most researches focus on the sampling effect associated with the random sampling from a population of raindrops. The effect of sampling variation on raindrop size measurements in stationary rainfall by using a stochastic model was researched by Uijlenhoet et al. [14], the sampling effects in DSD measurements in nonstationary rain were simulated by Berne and Uijlenhoet [15], and the sampling uncertainty associated with OTT PARSIVEL disdrometer has been investigated by Jaffrain and Berne [16]; for the latter, different instruments have varied parameters, such as the sampling area, sampling rate, and bin sizes and widths; they also influence the accuracy of measurement and reconstruction of precipitation distribution fundamentally. Marzuki et al. investigated the effect of binning on the DSD parameter estimates from 2DVD measurement and found that the bin width selection influences the shape of DSD [17]. Checa-Garcia et al. investigated the binning effects on drop size distribution (DSD) 
measurements obtained by JWD, POSS, Thies, PARSIVEL, 2DVD, and optical spectropluviometer instruments and found that the instrument differences due to sampling were a relevant uncertainty, but concentration, reflectivity, and massweighted diameter were sensitive to binning [18].

The choice of bin size classes may influence the shape of the DSD fundamentally; a too large bin size may ignore certain small sizes of drops, resulting in that the measured DSD would not represent the underlying DSD; and the bin size cannot be small infinitely because of the limitation of the instrument's resolution. Furthermore, different bin size classes may cause different representative diameters in the average and integral processes, which influence the results of moments of the DSD; different bin sizes exhibit different scaling relations with respect to the number of samples, sequences, and amplitude of rain-rate. Therefore, the optimal widths of the bin for a certain instrument should be investigated and handled carefully.

We have developed a precipitation microphysical characteristic sensor (short for PMCS, called Video Precipitation Sensor before) based on particle image velocimetry techniques recently [19]; it can measure the size, shape, and fall velocity of hydrometeors from a CMOS camera illuminated by a pulsed LED light source. In order to quantify the sampling effect of different bin sizes on the DSD parameters measured by PMCS, we simulate a sampling process of raindrops by PMCS based on a particle-by-particle Monte-Carlo model; the effects of different sampling bin sizes on DSD measurement are discussed, and the optimum sampling bin size and width for PMCS are proposed; at last the field measurement of a PMCS, an OTT PARSIVEL disdrometer, and a tipping bucket rain Gauge was carried out, and the rain-rate, rainfall accumulation, and DSD related parameters are compared, by which the performance of PMCS with specific sampling parameters is validated.

\section{Precipitation Microphysical Characteristics Sensor (PMCS)}

The PMCS consists of four units: optical unit, imaging unit, acquisition and control unit, and data processing unit, as shown in Figure 1(a). The optical unit (OU) contains a light-emitting diode (LED), a multimode fibre cluster, expanded beam lens, and concentration lens, which can provide a parallel cylindrical light beam for imaging unit; the imaging unit (IU) contains one complementary metal oxide semiconductor (CMOS) image sensor and driving circuit, which record digital images with 640 pixels $\times 480$ pixels and 50 frames per second (fps), and the sampling volume is $300 \mathrm{~mm} \times 40 \mathrm{~mm} \times 30 \mathrm{~mm}$; hence the pixel size of an image is $0.0625 \mathrm{~mm}$; the acquisition and control unit contains a digital signal processor (DSP) and field programmable gate array (FPGA), which generates and outputs timing signals of double exposures in one frame, controls the optical unit and imaging unit, records and preprocesses the raw images, and encodes and transmits the raw data; the data processing unit (DPU) is a PC terminal that communicates with the acquisition and control unit using a coaxial network cable via the Internet TCP/IP protocol. Software running on the terminal receives the data obtained by the sensor, processes the hydrometeors' images, and calculates the size, velocity, and shape of hydrometeors. A photography of the PMCS is as shown in Figure 1(b); the optical unit and imaging unit are integrated into one tunnel housing, on which there are a couple of metal splash elimination grids, to prevent raindrops splashing into the sampling area and to minimize the wind disturbances about the instruments.

The double-exposure in one frame (DEOF) of the PMCS plays a key role in the simultaneous measurement of the size, shape, and fall velocity of precipitation particles, as shown in Figure 2. The CMOS camera runs in 50 frames per second, a pulse synchronous signal generator is used to generate two exposures from pulse light source in each frame, the single exposure time is $20 \mu \mathrm{s}$, and the interval of the two exposures is $2 \mathrm{~ms}$. The CMOS camera captures the double-exposure image of each particle in a single frame, by which the particle shape information can be obtained, and the size, axis ratio, and canting angle of particles can be calculated, and the velocity can be calculated according to the displacement and time internal.

\section{Simulation of Different Sampling Bin Sizes}

In fact, fluctuations in DSD measurements and derived rainfall properties are due to not only the real fine-scale physical variations (called natural variability), but also the statistical sampling errors (called sampling fluctuations) [20]. The natural variability is associated with the actual rainfall variation in the natural environment, depending on the local weather condition, rainfall type, and its fine-scale variation, whereas the sampling fluctuation is associated with the instrument itself, depending on the sampling area, bin sizes, resolution, and other parameters. Given that different instruments have different parameters and rain-rate is integrated by the diameter and fall velocity of raindrops with different numbers in different bins, there might be certain discrepancies in the output of different instruments during the same rainfall event, especially for the higher rain-rate.

Considering the operation principle of PMCS, the effective sampling time is only $10 \%$ of the exposure time of each frame; not all particles passing through the sampling area can be double-imaged by camera; the capture probability of a certain particle which can be fully photographed twice by the camera decreases exponentially with its size. Considering this measurement mechanism, the measured DSD might deviate with the real DSD due to unreasonable parameters settings. Therefore, the uncertainty in DSD measured by PMCS with different sampling parameters should be evaluated, and the optimum sampling bin sizes of PMCS should be researched and proposed.

3.1. Methodology. The sampling processes of raindrops by PMCS can be simulated based on a particle-by-particle Monte-Carlo model; the simulation contains (1) marked point process of raindrops arriving at the sampling volume; (2) probabilistic capture process of double-exposure images of raindrops recorded by PMCS; (3) DSD inversion process 


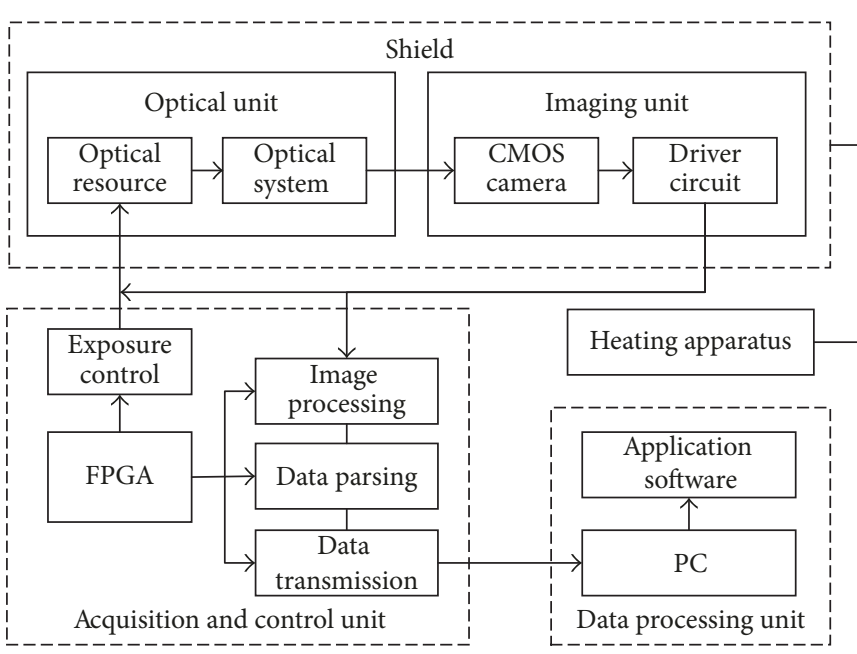

(a)

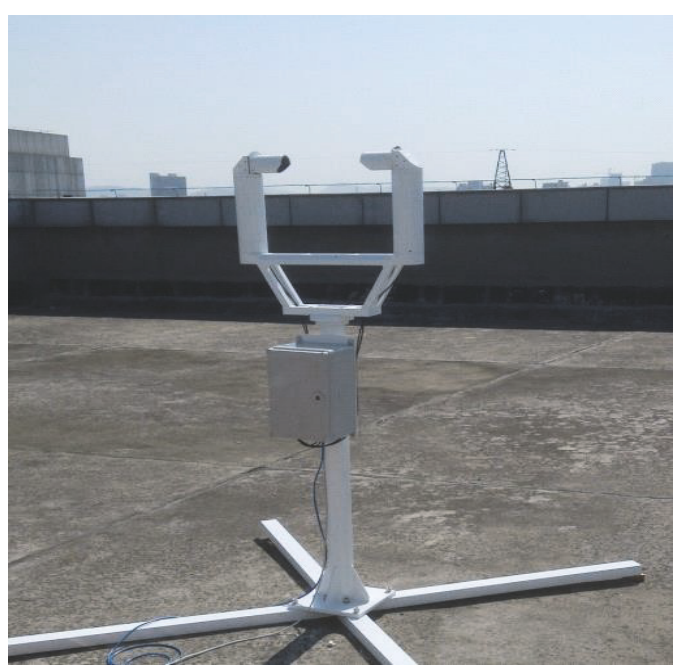

(b)

FIGURE 1: The framework of precipitation microphysical characteristic sensor.
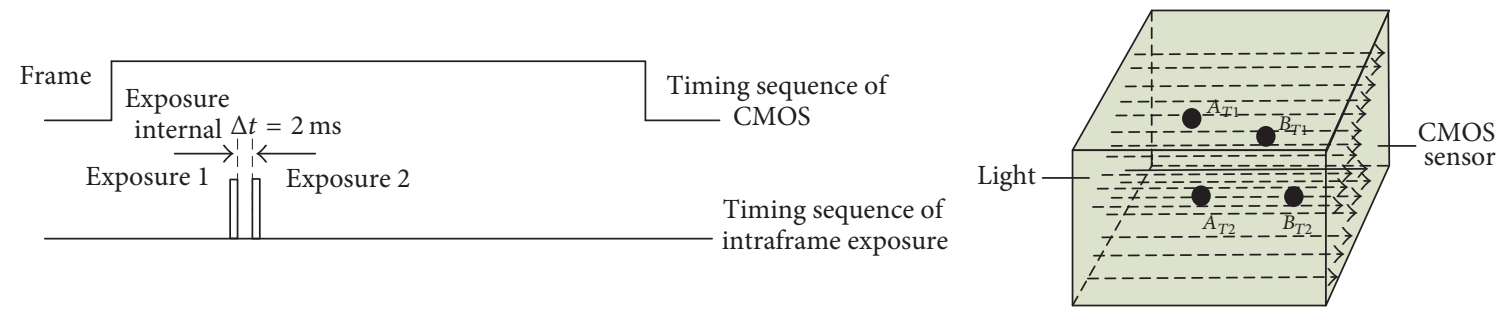

FIGURE 2: The principle of measurement.

of calculation of raindrop size distribution from sampling volume to unit volume. The details are described as follows.

3.1.1. Marked Point Process. Assuming that the positions of raindrops arriving at the sampling area in space follow the homogeneous Poisson model [21], the diameter and number of raindrops yields to the probabilistic model of raindrop size distribution, the terminal velocity of raindrops in the air depends exclusively on their diameter, and raindrops do not interact with each other. The time, number, and size of raindrops sampled by PMCS are simulated based on the above assumption.

The arriving time $(\mathrm{s})$ and diameter $(\mathrm{mm})$ of $i$ th raindrops are denoted as $T_{i}$ and $D_{i}$; the total number of raindrops arriving at the sampling volume for the $i$ th arrival time which is less than or equal to $t$ is

$$
\begin{gathered}
\eta(t)=\sum_{i=1}^{\infty} l\left(T_{i} \leq t\right), \\
l\left(T_{i} \leq t\right)= \begin{cases}1, & \text { if } T_{i} \leq t \\
0, & \text { if } T_{i}>t,\end{cases}
\end{gathered}
$$

where the time origin is arbitrary and it can represent the beginning of a storm and the beginning of a minute, hour, day, and so forth [21]. The time origin typically represents the beginning of a rainfall period, $t$ is 1 minute in the following simulation, and the unit is second. Note that $\eta(t)$ sums from 1 to infinity to include as many raindrops as possible but does not imply that there will be infinite raindrops in a certain time interval.

Assuming that $\lambda(t)$ is the mean number of raindrops arrivals at the top of sampling volume at time $t$ (drops per square meter per second), $\theta(t)$ is the parameters of DSD at time $t$. Raindrops arrive at the upper surface of sampling volume according to a Poisson process with time-varying occurrence $\lambda(t)$; the DSD is given by a probability density function $f[y \mid \theta(t)]$. The corresponding probability of raindrops which have number $\eta(t)=k(k=0,1,2, \ldots)$ can be written as follows:

$$
\begin{aligned}
P[ & {[\eta(t)=k \mid \lambda(u), \theta(u) ; u \leq t] } \\
& =\frac{1}{k !}\left[\int_{o}^{t} \lambda(u) d u\right]^{k} \exp \left[-\int_{o}^{t} \lambda(u) d u\right] .
\end{aligned}
$$

Here we use $N_{0}, \mu, \Lambda$ of Gamma function for DSD parameters, and the diameter $D_{i}(\mathrm{~mm})$ of corresponding raindrops at time $t$ (s) can be obtained from the probabilistic model of Gamma function:

$$
P\left[D_{i} \leq x \mid \lambda(u), \theta(u) ; u \leq t\right]=\int_{o}^{x} f\left[y \mid \theta\left(t_{i}\right)\right] d y,
$$


where $f[y \mid \theta(t)]=N_{0}(t) \cdot y^{\mu(t)} \cdot \exp (-\Lambda(t) y), x(\mathrm{~mm})$ is the maximum diameter of raindrops, and $y(\mathrm{~mm})$ is the diameter of raindrops.

3.1.2. Probabilistic Capture Process. Consider that not all particles that pass through the sampling volume can be doubleimaged by PMCS. The probability that a certain raindrop with a certain size and fall velocity can be fully photographed at least once by the camera in the vertical dimension is defined as Prob ${ }_{\text {vertical }}$ :

$$
\begin{aligned}
\text { Prob }_{\text {vertical }} & =\frac{H-D_{v}-V(D) T_{\text {interval }}}{V(D) \cdot 20 \mathrm{~ms}}, \\
V(D) & =9.65-10.3 \exp (-0.6 D),
\end{aligned}
$$

where $H$ is the height of sampling volume (30 $\mathrm{mm}$ for PMCS), $D_{V}$ is the vertical size of raindrops $(\mathrm{mm}), V(D)$ is the fall velocity of raindrops $(\mathrm{m} / \mathrm{s})$ associated with the diameter of raindrops, $T_{\text {interval }}$ is the time interval between two exposures in each frame $(2 \mathrm{~ms})$, and $20 \mathrm{~ms}$ is the total exposure time of each frame.

Whether raindrops passing through the sampling volume can be fully photographed twice in a single frame can be estimated as follows:

$$
\text { flag }\left(T_{i}, D_{i}\right)= \begin{cases}1, & \text { if } r \leq \operatorname{Prob}_{\text {vertical }}\left(D_{i}\right) \\ 0, & \text { if } r>\operatorname{Prob}_{\text {vertical }}\left(D_{i}\right),\end{cases}
$$

where $r$ is a random number between 0 and 1 generated by uniform probability model. When $r$ is equal to or less than the capture probability of one raindrop with diameter $D$, this raindrop is labeled as captured, otherwise it is labeled as uncaptured.

3.1.3. DSD Inversion Process. The total number of raindrops can be calculated as follows:

$$
\operatorname{Num}(D)=\operatorname{Num}_{\text {single }}(D)+\frac{\operatorname{Num}_{\text {double }}(D)}{2},
$$

where $\operatorname{Num}_{\text {single }}(D)$ is the number of raindrops captured by only one exposure in a single frame and $\operatorname{Num}_{\text {double }}(D)$ is the number of raindrops captured by double exposures in a single frame.

According to (4) and (6), the number of raindrops per unit volume $\left(\mathrm{m}^{3}\right)$ and per unit diameter $(\mathrm{mm})$ can be calculated as follows:

$$
n(D)=\frac{\operatorname{Num}(D)}{A_{\text {sampling }} \cdot d D \cdot \text { Prob }_{\text {vertical }}},
$$

where $A_{\text {sampling }}=300 \mathrm{~mm} \times 40 \mathrm{~mm} \times 30 \mathrm{~mm}$. Considering that the raindrops of different sizes have different fall velocities, the velocity correction algorithm is adopted to obtain the number of raindrops per unit volume $\left(\mathrm{m}^{3}\right)$ and per unit size class $(\mathrm{mm})$ :

$$
\begin{aligned}
& N(D)=\frac{n(D)}{V(D) \times t}, \\
& V(D)=9.65-10.3 \exp (-0.6 D) .
\end{aligned}
$$

DSD characterization consists of mass-weighed mean diameter $D_{m}(\mathrm{~mm})$ and standard deviation of mass-weighed spectrum $\sigma_{m}(\mathrm{~mm})$, as shown in (9). $D_{m}$ is defined as the ratio of mass-weighed diameter and total mass of all raindrops in unit volume, which denotes the average of DSD. $\sigma_{m}$ is defined as the deviation of the diameter with the $D_{m}$ of all raindrops, which denotes the variance of DSD.

$$
\begin{aligned}
D_{m} & =\frac{\int_{0}^{\infty} N(D) D^{4} d D}{\int_{0}^{\infty} N(D) D^{3} d D}, \\
\sigma_{m}^{2} & =\frac{\int_{0}^{\infty} N(D)\left(D-D_{m}\right)^{2} D^{3} d D}{\int_{0}^{\infty} N(D) D^{3} d D} .
\end{aligned}
$$

The rain-rate $R(\mathrm{~mm} / \mathrm{h})$ and radar reflectivity factor $Z(\mathrm{~dB})$ can be integrated from $N(D)$ :

$$
\begin{aligned}
& R=\frac{\pi}{6} \int_{0}^{\infty} N(D) \rho D^{3} V(D) d D, \\
& Z=\int_{0}^{\infty} N(D) D^{6} d D .
\end{aligned}
$$

The Gamma function $N(D)$ of DSD is defined as follows:

$$
N(D)=N_{0} \cdot D^{\mu} \cdot \exp (-\Lambda D),
$$

where $N_{0}$ is the intercept parameter $\left(\mathrm{m}^{-3} \mathrm{~mm}^{-1-\mu}\right), \mu$ is the shape parameter, and $\lambda$ is the slope of the distribution $\left(\mathrm{mm}^{-1}\right)$; they can be calculated by $2 \mathrm{nd}, 3 \mathrm{rd}$, and 4 th moments as follows:

$$
\begin{aligned}
N_{0} & =\frac{(\mu+4)^{\mu+4}}{(\mu+3) !} \cdot\left(\frac{M_{3}}{M_{4}}\right)^{\mu+4} \cdot M_{3}, \\
\mu & =\frac{\left(3 M_{4} M_{2}-4 M_{3}^{2}\right)}{\left(M_{3}^{2}-M_{4} M_{2}\right)}, \\
\Lambda & =(\mu+4) \frac{M_{3}}{M_{4}} .
\end{aligned}
$$

The $n$th moment is defined as follows:

$$
M_{n}=\int_{0}^{\infty} N(D) D^{n} d D .
$$

3.2. Parameters Setting. The simulation parameters consist of raindrop size distribution, sampling time, sampling volume, and bin size and width. The former is related to the natural precipitation itself; the latter three parameters are related to the instrument, in which the sampling time and sampling volume are fixed; considering the effect of capture probability on DSD measurement of PMCS under different rainfall conditions, we focus on the optimization of sampling bin size and width.

Table 1 shows the sampling bin size of existing disdrometers; these settings are based on the size resolution of different disdrometers, JWD has the least bin numbers (20), and the intervals range from $0.09 \mathrm{~mm}$ to $0.57 \mathrm{~mm}$. This disdrometer 
TABLE 1: Sampling bin size of different disdrometers.

\begin{tabular}{lcc}
\hline Disdrometer & Bin size $[\mathrm{mm}]$ & Bin number \\
\hline OTT PARSIVEL & $0.125,0.25,0.375,0.5,0.625,0.75,0.875,1.0,1.125,1.25,1.5,1.75,2,2.25,2.5,3.0,3.5$, \\
disdrometer (OTT) & $4,4.5,5,6,7,8,9,10,12,14,16,18,20,23,26$ & 32 \\
2D video disdrometer & $0.2,0.4,0.6,0.8,1.0,1.2,1.4,1.6,1.8,2.0,2.2,2.4,2.6, \ldots, 9.6,9.8,10$ & 50 \\
(2DVD) & $0.313,0.405,0.505,0.596,0.715,0.827,0.999,1.232,1.429,1.582,1.748,2.077$, \\
Joss-Waldvogel & $2.441,2.727,3.011,3.385,3.704,4.127,4.573,5.145$ & 20 \\
disdrometer (JWD) & $0.25,0.375,0.5,0.75,1.0,1.25,1.5,1.75,2.0,2.5,3.0,3.5,4.0,4.5,5.0,5.5,6.0,6.5,7.0$, \\
Thies disdrometer (Thies) & $7.5,8.0$ & 22 \\
\hline
\end{tabular}

has 22 bin numbers and the intervals are $0.125 \mathrm{~mm}, 0.25 \mathrm{~mm}$, and $0.5 \mathrm{~mm}$ separately; OTT disdrometer has 32 bin numbers and the intervals range from $0.125 \mathrm{~mm}$ to $3 \mathrm{~mm}$. The above three disdrometers have uninform intervals, whereas 2DVD has a nominal resolution of $0.2 \mathrm{~mm}$; the bin size can be determined in postprocessing by the user; it has a maximum bin number of 50 with a uniform interval $(0.2 \mathrm{~mm})$. The raw size resolution of PMCS is $0.1 \mathrm{~mm}$, and the question is whether the raw size resolution can be directly adopted as the best bin size.

Taking the above setting modes of bin size and width as references, we propose four schemes of bin sizes for PMCS, as shown in Table 2. The raw output of PMCS ranges from $0.1 \mathrm{~mm}$ to $7.0 \mathrm{~mm}$, which has 70 bin numbers with $0.1 \mathrm{~mm}$ interval; intervals $1,2,3$, and 4 denote four schemes of bin sizes, having $25,35,26$, and 25 bin numbers separately.

Rain-rate can be seen as the macroscopic expression of the raindrop size distribution; the rainfalls are usually categorized into very light, light, moderate, heavy, very heavy, and extreme rainfalls according to their rain-rate [22]. Considering that the rain-rate $R$ can be calculated by integration of the parameters of Gamma functions $N_{0}, \mu$, and $\Lambda$, four typical rain-rate categories of rainfall are chosen to simulate the sampling process of PMCS; the parameters are shown in Table 3.

3.3. Results. Based on the above simulation parameters, we simulate the sampling process of PMCS with a specific DSD in a steady rainfall event, and the rain-rate categories are set to $1.4 \mathrm{~mm} / \mathrm{h}, 7.28 \mathrm{~mm} / \mathrm{h}, 15.81 \mathrm{~mm} / \mathrm{h}$, and $58.58 \mathrm{~mm} / \mathrm{h}$ separately, which is used to evaluate the sampling effect of PMCS in different rain-rate categories. The simulation results of raindrop size distribution are shown in Figure 3. The real DSD is the precondition for the simulation, the raw capture DSD is measured by PMCS directly, without sorting raindrops into bins and correction of capture probability, and intervals 1-4 are the DSDs calculated after correction of capture probability based on the sortation of raindrops into four different bins. The S, M, and L in Figure 3 denote size range for small raindrops, median raindrops, and large raindrops separately; it should be noted that the size ranges correspond to different diameters in different rain-rate categories. Generally speaking, the raw capture DSD is lower than the inversed DSDs from 4 intervals obviously due to the capture probability of PMCS, whereas the 4 inversed DSDs agree well with the real DSDs for the small raindrops, and there are some differences for the median and large raindrops.
For the small raindrops, the raw DSD and inversed DSDs from interval 1, interval 3, and interval 4 agree well with the real DSDs, while the inversed DSDs from interval 2 are lower than the real DSD due to the larger intervals between bin sizes in the small-size range. The median raindrops have the largest number density, which contribute most to rain-rate; the number density and width of median raindrops increase with the increasing of rain-rate, and the raw capture DSD is obviously lower than the real DSD because of the unsteady capture probability, which takes on a nonuniform step downward trend, especially in the light rainfall and rainstorm; there is no significant discrepancy between the inversed DSDs from interval 1, interval 2, interval 3, and interval 4, which agree well with real DSD. The number density of large raindrops determines the final shape of DSD, influenced by the capture probability, the raw capture DSD has a significant fluctuation variation, and a certain number of large raindrops are missed; compared with the raw capture DSD, there are good consistencies between the inversed DSDs from 4 interval schemes and real DSD; the reason is that the appropriate sampling bin size and width can reduce the impact of variation of raindrop number on DSD shape. It should be noted that there are also some certain deviations between the inversed DSD and real DSD, especially for the inversed DSDs from interval 3 in the light rainfall and from interval 2 in the rainstorm.

Considering the different consistencies between the inversed DSDs and the real DSD, each sampling process is simulated 1,000 times, and about 35,000, 86,000, 102,000, and 222,000 raindrops are simulated for 4 rain-rate categories separately. Then the relative deviation $\varepsilon_{X}$ of variable is adopted to evaluate the accuracy of inversed DSDs by different sampling schemes quantitatively. The expression can be written as follows:

$$
\varepsilon_{X}=\frac{1}{n} \sum_{i=1}^{n}\left|\frac{X_{i}-X_{0}}{X_{0}}\right|,
$$

in which $X_{i}$ is the simulation results of $i$ th time, $X_{i}$ is the real value of parameters, and $n$ denotes the total number of simulations.

The simulation results of $D_{m}, \sigma_{m}, R$, and $Z$ are shown in Figure 4 . It can be seen that the mean and standard deviation of relative error decrease with the increase of rain-rate overall; the least means of $\varepsilon_{D_{m}}$ and $\varepsilon_{\sigma_{m}}$ are less than $2 \%$; the relative error of $Z$ has the largest mean and standard deviation, about $5 \%$ and $13 \%$ separately. For $D_{m}$, the overall mean of $\varepsilon_{D_{m}}$ from interval 4 has the least value $0.72 \%$, and the mean of 
TABLE 2: Four schemes of sampling bin size for PMCS.

\begin{tabular}{lcc}
\hline Scheme & Bin size $[\mathrm{mm}]$ & Bin number \\
\hline Raw output & $0.1,0.2,0.3,0.4,0.5,0.6,0.7,0.8,0.9,1.0, \ldots, 6.3,6.4,6.5,6.6,6.7,6.8,6.9,7.0$ & 70 \\
Interval 1 & $4.8,5.6,6.4,7.0$ & 25 \\
& $0.1,0.2,0.3,0.4,0.5,0.6,0.7,0.8,1.0,1.2,1.4,1.6,1.8,2.0,2.2,2.4,2.8,3.2,3.6,4.0,4.4$, & 35 \\
Interval 2 & $0.2,0.4,0.6,0.8,1.0,1.2,1.4,1.6,1.8,2.0,2.2,2.4,2.6,2.8,3.0,3.2,3.4,3.6,3.8,4.0,4.2$, \\
& $4.4,4.6,4.8,5.0, \ldots, 6.0,6.2,6.4,6.8,7.0$ & 26 \\
Interval 3 & $4.1,0.2,0.3,0.4,0.5,0.6,0.7,0.8,1.0,1.2,1.4,1.6,1.8,2.1,2.4,2.7,3.0,3.3,3.6,3.9,4.2$, \\
& $4.0,5.6,6.2,7.0$ & 25 \\
Interval 4 & $5.1,0.2,0.3,0.4,0.5,0.7,0.9,1.1,1.3,1.5,1.7,1.9,2.1,2.5,2.9,3.3,3.7,4.1,4.5,4.9,5.3$, & 2.0 \\
\hline
\end{tabular}

TABLE 3: The Gamma raindrop size distribution parameters for different rain-rate categories.

\begin{tabular}{lcccc}
\hline Category & $N_{0}\left[\mathrm{~m}^{-3} \mathrm{~mm}^{-1}\right]$ & $\mu$ & $\Lambda\left[\mathrm{mm}^{-1}\right]$ & Rain-rate $\left[\mathrm{mm} \mathrm{h}^{-1}\right]$ \\
\hline Light rain & $1.31 \times 10^{4}$ & 2.3 & 4.7 & 1.4 \\
Moderate rain & $8.01 \times 10^{4}$ & 3.9 & 5.2 & 7.3 \\
Heavy rain & $3.32 \times 10^{5}$ & 6.1 & 6.3 & 15.8 \\
Rainstorm & $2.85 \times 10^{4}$ & 3.24 & 3.38 & 55.6 \\
\hline
\end{tabular}
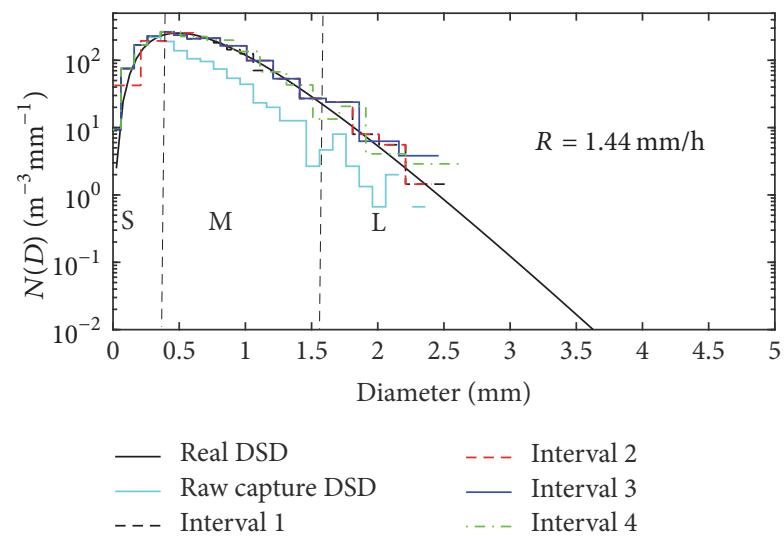

(a)

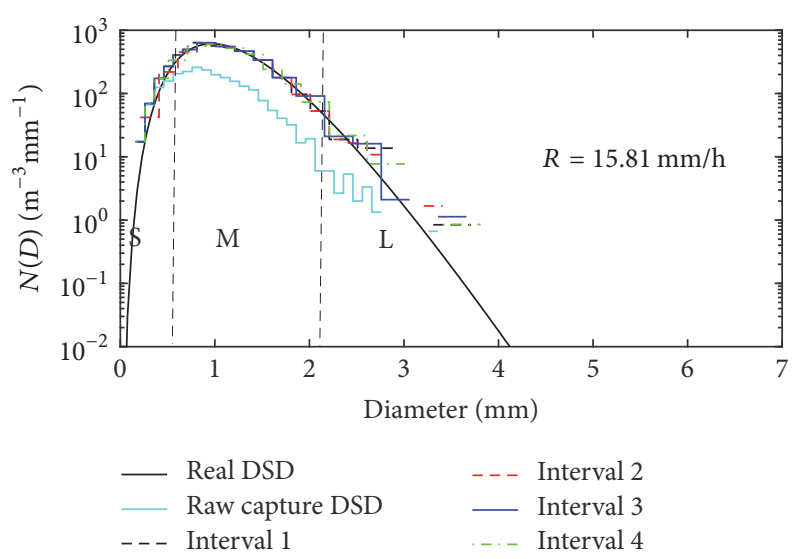

(c)

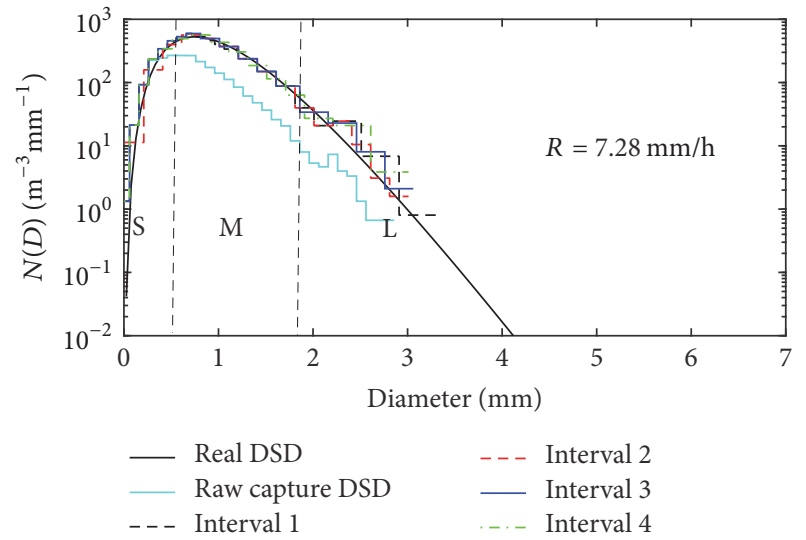

(b)

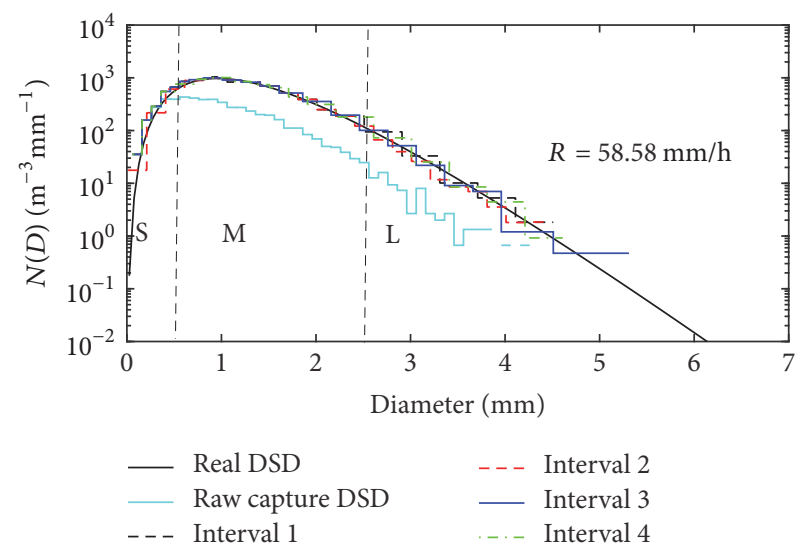

(d)

FIGURE 3: Real DSD and inversed DSDs under the condition of different rain-rate categories. 


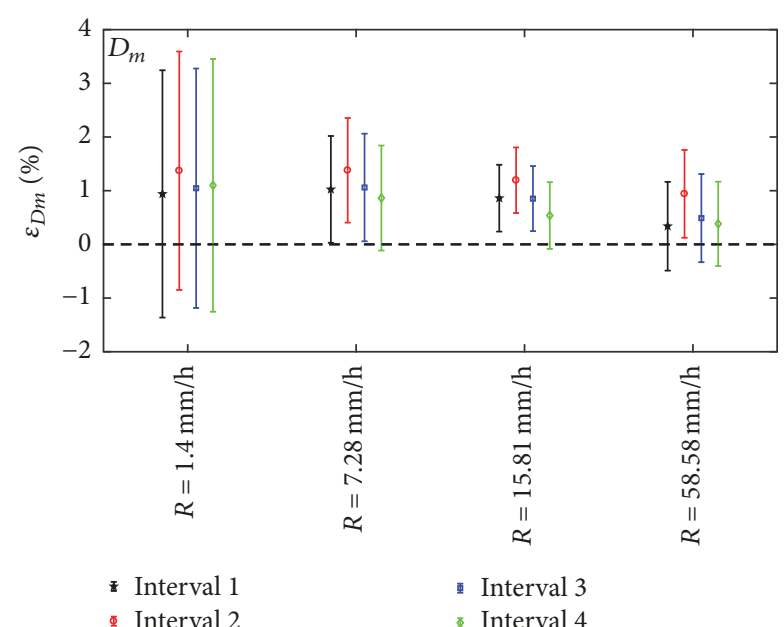

(a)

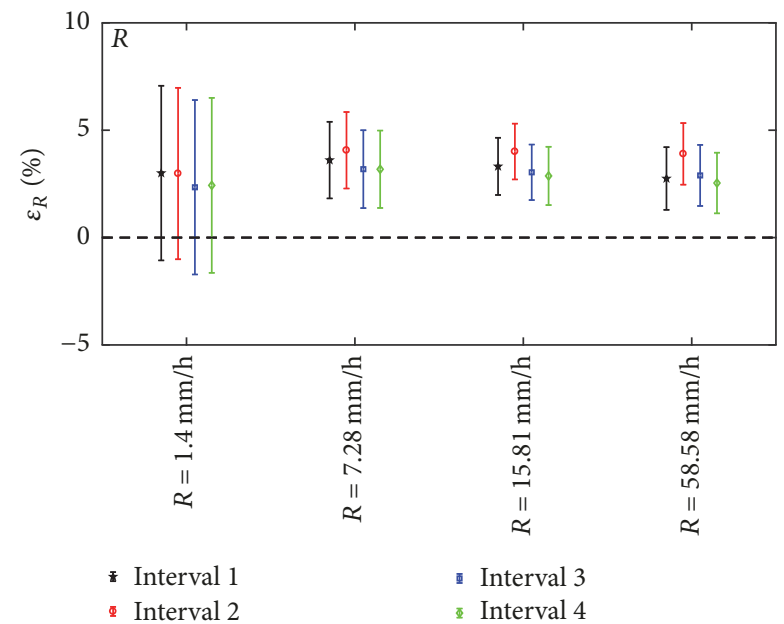

(c)

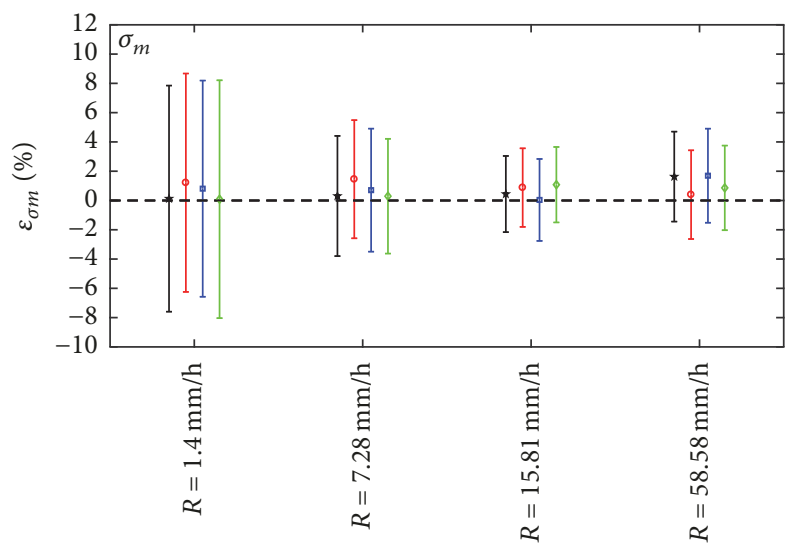

* Interval 1

Interval 2

Interval 3

Interval 4

(b)

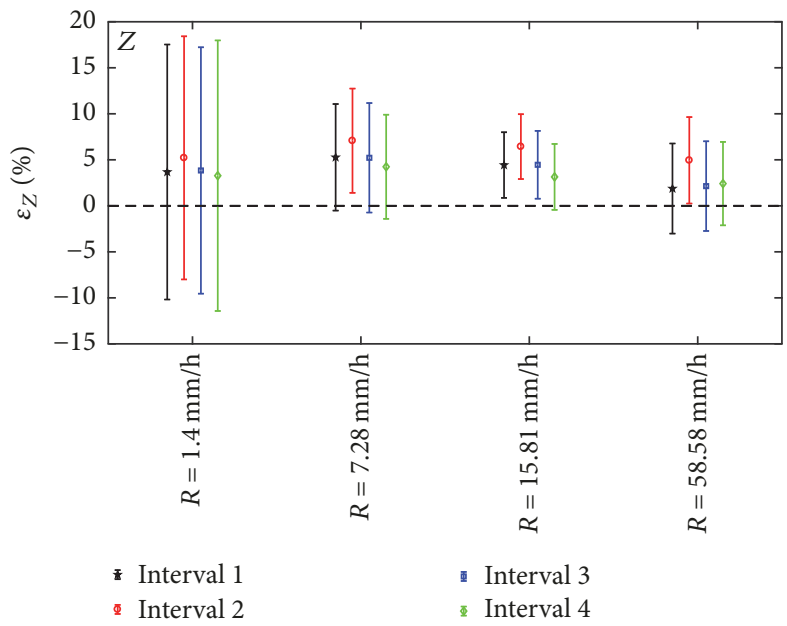

(d)

FIGURE 4: The mean and standard deviation of relative error of $D_{m}, \sigma_{m}, R$, and $Z$.

$\varepsilon_{D_{m}}$ from interval 1 in the light rain and heavy rain has the least value. For $\sigma_{m}$, the least means of $\varepsilon_{\sigma_{m}}$ are $0.09 \%, 0.29 \%$, $0.04 \%$, and $0.40 \%$ from interval 4 in the light rainfall and moderate rainfall, from interval 3 in the heavy rainfall, and from interval 2 in the rainstorm. For $R$, the overall mean of $\varepsilon_{R}$ is $3.27 \%$, and four interval schemes have good performances in the moderate rainfall, heavy rainfall, and rainstorm, except that $\varepsilon_{R}$ has a larger standard deviation in the light rainfall, which means that it has a wider variation. For $Z$, interval 4 has the best performance in the light rainfall, moderate rainfall, and heavy rainfall; the mean of $\varepsilon_{Z}$ is $3.27 \%, 4.24 \%$, and $3.14 \%$ separately, whereas interval 1 has the best performance in the rainstorm, and the mean of $\varepsilon_{Z}$ is $1.88 \%$.

The simulation results of $N_{0}, \mu$, and $\Lambda$ of Gamma function are shown in Figure 5. It can be seen that the mean and standard deviation of $N_{0}$ decrease with the increase of rainrate, $N_{0}$ has the greatest error in the light rainfall, and the maximum mean of relative error of $N_{0}$ is $40.52 \%$, whereas $\mu$ and $\Lambda$ have less relative error, and their error increases with the increase of rain-rate. $N_{0}$ have the least relative error from interval 3 in light rainfall, interval 4 in moderate rainfall, interval 3 in heavy rainfall, and interval 2 in rainstorm separately; the minimum means of $\varepsilon_{N_{0}}$ are $28.72 \%, 2.36 \%$, $2.94 \%$, and $0.77 \%$; $\mu$ have the least relative error from interval 1 in light rainfall, interval 3 in moderate rainfall, interval 2 in heavy rainfall, and interval 4 in rainstorm separately; the minimum means of $\varepsilon_{\mu}$ are $0.87 \%, 0.41 \%, 0.83 \%$, and $4.76 \%$; $\Lambda$ have the least relative error from interval 1 in light rainfall and moderate rainfall, interval 3 in heavy rainfall, and interval 2 in rainstorm separately; the minimum means of $\varepsilon_{\Lambda}$ are $0.94 \%$, $0.11 \%, 0.53 \%$, and $1.33 \%$.

Table 4 lists the mean relative error of the above parameters; it can be seen that four schemes of sampling intervals have different performances on raindrop size distribution parameters in four rain-rate categories. For $D_{m}, \sigma_{m}, R, Z$, $N_{0}, \mu$, and $\Lambda$, the scheme interval 2 has the maximum mean relative error in light rainfall, moderate rainfall, and heavy rainfall $(8.10 \%, 5.38 \%$, and $3.62 \%)$, and the scheme interval 4 has the minimum mean relative error in light rainfall, moderate rainfall, and rainstorm $(5.40 \%, 2.19 \%$, and $2.25 \%)$; and for the average of four rain-rate categories, the mean relative errors of the scheme interval 1, interval 2, interval 3, 


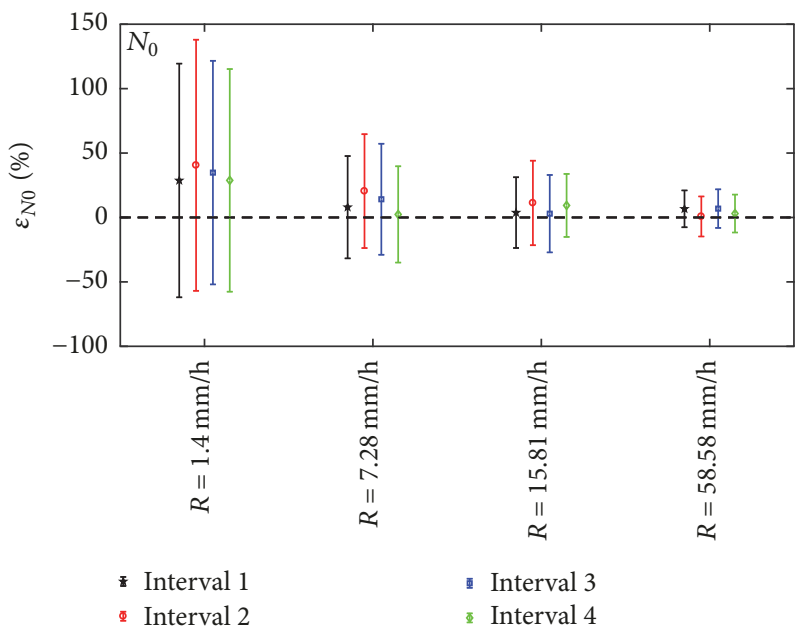

(a)

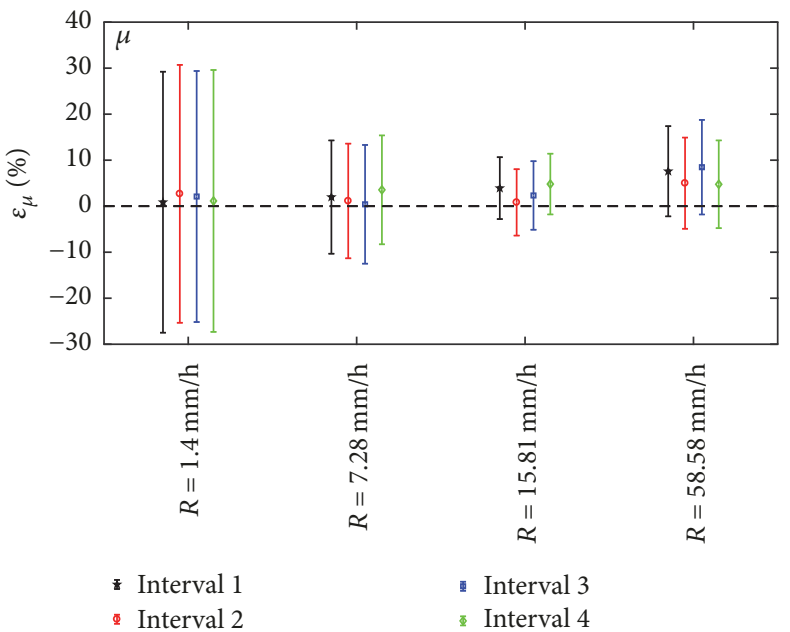

(b)

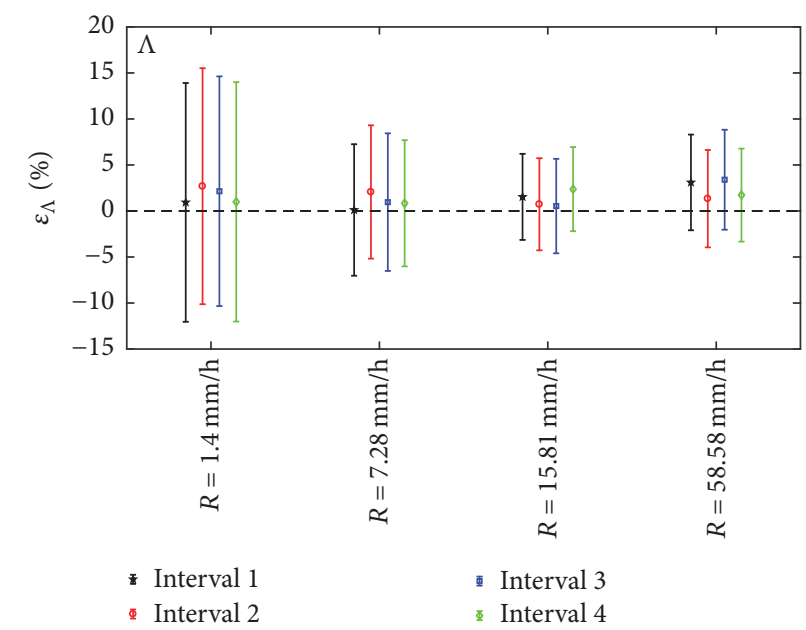

(c)

FIgURE 5: The mean and standard deviation of relative error of $N_{0}, \mu$, and $\Lambda$.

TABLE 4: Mean relative error of parameters in different rain-rate categories.

\begin{tabular}{|c|c|c|c|c|c|c|c|c|}
\hline$R[\mathrm{~mm} / \mathrm{h}]$ & Scheme & $D_{m}[\%]$ & $\sigma_{m}[\%]$ & $R$ [\%] & $Z$ [\%] & $N_{0}[\%]$ & $\mu[\%]$ & $\Lambda[\%]$ \\
\hline \multirow{4}{*}{1.4} & Interval 1 & 0.94 & 0.13 & 3.00 & 3.67 & 28.72 & 0.87 & 0.94 \\
\hline & Interval 2 & 1.37 & 1.22 & 2.98 & 5.21 & 40.52 & 2.69 & 2.69 \\
\hline & Interval 3 & 1.05 & 0.81 & 2.34 & 3.84 & 34.81 & 2.10 & 2.15 \\
\hline & Interval 4 & 1.10 & 0.09 & 2.43 & 3.27 & 28.79 & 1.14 & 0.99 \\
\hline \multirow{4}{*}{7.28} & Interval 1 & 1.03 & 0.31 & 3.61 & 5.27 & 7.96 & 1.98 & 0.11 \\
\hline & Interval 2 & 1.38 & 1.45 & 4.07 & 7.08 & 20.45 & 1.14 & 2.07 \\
\hline & Interval 3 & 1.06 & 0.71 & 3.19 & 5.22 & 14.13 & 0.41 & 0.96 \\
\hline & Interval 4 & 0.86 & 0.29 & 3.18 & 4.24 & 2.36 & 3.55 & 0.83 \\
\hline \multirow{4}{*}{15.81} & Interval 1 & 0.86 & 0.44 & 3.32 & 4.43 & 3.77 & 3.92 & 1.54 \\
\hline & Interval 2 & 1.19 & 0.88 & 4.01 & 6.43 & 11.26 & 0.83 & 0.73 \\
\hline & Interval 3 & 0.85 & 0.04 & 3.04 & 4.46 & 2.94 & 2.33 & 0.53 \\
\hline & Interval 4 & 0.54 & 1.08 & 2.87 & 3.14 & 9.35 & 4.81 & 2.37 \\
\hline \multirow{4}{*}{58.58} & Interval 1 & 0.34 & 1.63 & 2.75 & 1.88 & 6.70 & 7.59 & 3.10 \\
\hline & Interval 2 & 0.94 & 0.40 & 3.90 & 4.95 & 0.77 & 5.00 & 1.33 \\
\hline & Interval 3 & 0.49 & 1.69 & 2.89 & 2.14 & 6.87 & 8.48 & 3.40 \\
\hline & Interval 4 & 0.38 & 0.86 & 2.54 & 2.41 & 3.05 & 4.76 & 1.73 \\
\hline
\end{tabular}


and interval 4 are $3.60 \%, 4.89 \%, 4.03 \%$, and $3.32 \%$ separately; it can be concluded that the scheme interval 4 has the best performance in the four categories of rainfall on the whole; therefore the scheme interval 4 is adopted for the optimal sampling bin sizes of PMCS.

The accuracy of DSD measurements varies with the different instruments with different sampling principles and different parameters settings, and there is no ideal reference of rainfall field to test and evaluate them. This method based on sampling process simulation can be applied not only for performance evaluation of existing instruments, but also for optimization of developing instruments. Given the above discussions, based on the scheme interval 4 applied in PMCS, the results of field experiments are shown as follows.

\section{Field Experiments}

A joint observation of PMCS, SL-3 tipping bucket rain Gauge (short for Gauge), and OTT PARSIVEL disdrometer (short for OTT) was launched at Nanjing, China, during June 2015; the rainfalls are mainly convective rainfalls when it is plum rain season, and rainfall observations on the 16th, 17th, 25th, 26th, 27th, 28th, 29th, and 30th of June are collected and discussed. The rain-rate resolution and time resolution of PMCS, Gauge, and OTT are $0.001 \mathrm{~mm} / \mathrm{h}$ and $1 \mathrm{~min}, 0.1 \mathrm{~mm}$ and $1 \mathrm{~min}$, and $0.001 \mathrm{~mm} / \mathrm{h}$ and $10 \mathrm{sec}$. For the convenience of comparative analysis, the data of three instruments are processed in the same time resolution of $1 \mathrm{~min}$, and only the rainfalls heavier than $0.1 \mathrm{~mm} / \mathrm{h}$ from PMCS and OTT are used; after that the rainfalls obtained by all three instruments have 3,618 minutes' samples.

4.1. Rain-Rate and Rainfall Accumulation. The rain-rate and rainfall accumulations observed by PMCS, OTT, and Gauge are as shown in Figure 6. It can be seen that the sequential variations of rain-rate observed by PMCS, OTT, and Gauge have a good consistency and the rain-rate series of PMCS and OTT have several sharp increases due to the fine resolution of rain-rate. The maximum rain-rates measured by PMCS and OTT are $139.6 \mathrm{~mm} / \mathrm{h}$ and $134.8 \mathrm{~mm} / \mathrm{h}$, whereas the maximum rain-rate measured by Gauge is only $108 \mathrm{~mm} / \mathrm{h}$ because of the limited resolution and sampling principle. It tends to underestimate the rain-rate in heavy rainfall events [12]. The total accumulations of rainfall measured by PMCS, OTT, and Gauge are $426.8 \mathrm{~mm}, 480.8 \mathrm{~mm}$, and $412.7 \mathrm{~mm}$ separately. Considering that the Gauge is usually taken as a reference, the rainfall accumulations observed by PMCS and Gauge have higher consistency than those by OTT and Gauge, which validates the performance of PMCS for macroscopic variables measurement.

In order to quantify the discrepancies between three instruments, the relative deviation bias and absolute deviation ab_bias are defined as follows:

$$
\begin{aligned}
\text { bias } & =\frac{(1 / n) \sum_{i=1}^{n}\left(x_{i}-y_{i}\right)}{\langle x, y\rangle}, \\
\text { ab_bias } & =\frac{(1 / n) \sum_{i=1}^{n}\left|x_{i}-y_{i}\right|}{\langle x, y\rangle},
\end{aligned}
$$

in which $\langle x, y\rangle=(1 / n) \sum_{i=1}^{n}\left(\left(x_{i}+y_{i}\right) / 2\right)$ and $x$ and $y$ denote the data series with length $n$. And a moving average of 3 minutes is used to process the raw data to reduce the effect of sampling stochastic noises on the differential analysis between each instrument.

Figure 7 shows the comparisons of $3 \mathrm{~min}$ average rainrate between three instruments. It can be found that there are good linear relationships between every two instruments, especially between PMCS and OTT; the correlation coefficient between PMCS and OTT, PMCS and Gauge, OTT and Gauge is $0.98,0.96$, and 0.99 separately. Rain-rate measured by both PMCS and OTT is higher than rain-rate measured by Gauge, the relative deviations are $4.5 \%$ and $15.0 \%$, in which absolute deviation between PMCS and Gauge is higher than that between OTT and Gauge, the possible reason is that the sampling efficiency is affected by capture probability of PMCS, and the number density of large raindrops has an unsteady step-down variance.

In fact, rain-rates measured by different instruments have certain discrepancies in different rain-rate categories, as shown in Table 5. The absolute deviations of three instruments decrease with the increase of rain-rate on the whole. The relative deviations between OTT and PMCS are positive, which decreases with the increase of rain-rate; the relative deviations between PMCS and Gauge of $R<5 \mathrm{~mm} / \mathrm{h}$ and $R \geq$ $20 \mathrm{~mm} / \mathrm{h}$ are positive, and the relative deviations between PMCS and Gauge of $5 \leq R<10 \mathrm{~mm} / \mathrm{h}$ and $10 \leq R<20 \mathrm{~mm} / \mathrm{h}$ are negative; the relative deviations between OTT and Gauge are all positive, in which the relative deviations of $R<$ $5 \mathrm{~mm} / \mathrm{h}$ and $R \geq 20 \mathrm{~mm} / \mathrm{h}$ are higher than those of $5 \leq R<$ $10 \mathrm{~mm} / \mathrm{h}$ and $10 \leq R<20 \mathrm{~mm} / \mathrm{h}$. There is a good agreement between PMCS and OTT in all rain-rate categories; the correlation coefficient is about 0.9 , except for the correlation coefficient of 0.74 in $5 \leq R<10 \mathrm{~mm} / \mathrm{h}$. Above all, the rainrate and rainfall accumulations measured by PMCS agree well with the results measured by OTT and Gauge; the fluctuation variation with time may increase the absolute deviation, but the relative deviation remains small; it can be concluded that the performance of PMCS for macroscopic variables measurement is basically consistent with that of OTT and Gauge.

4.2. Raindrop Size Distribution. The raindrops size distribution can be described by mass-weighed mean diameter $D_{m}$ and standard deviation of mass-weighed spectrum $\sigma_{m}$, which denote the mean and variance of the spectrum. The DSDs measured by PMCS and OTT are processed by (9); the results are shown in Figure 8. $D_{m}$ and $\sigma_{m}$ obtained by PMCS and OTT have good accordance, the correlation coefficients are 0.9032 and 0.8261 , and the absolute deviations of $D_{m}$ and $\sigma_{m}$ between PMCS and OTT are $0.12 \mathrm{~mm}$ and $0.29 \mathrm{~mm}$ separately. The scatter plot shows that the discreteness of both $D_{m}$ and $\sigma_{m}$ increases with the increase of their values, and the discreteness of $\sigma_{m}$ is greater than that of $D_{m}$; the discreteness of both $D_{m}$ and $\sigma_{m}$ measured by OTT is greater than that measured by PMCS, denoting that PMCS can obtain a narrower spectral width of DSD than OTT.

In order to explore the discrepancy of DSD measured by PMCS and OTT thoroughly, the DSD data are divided into 


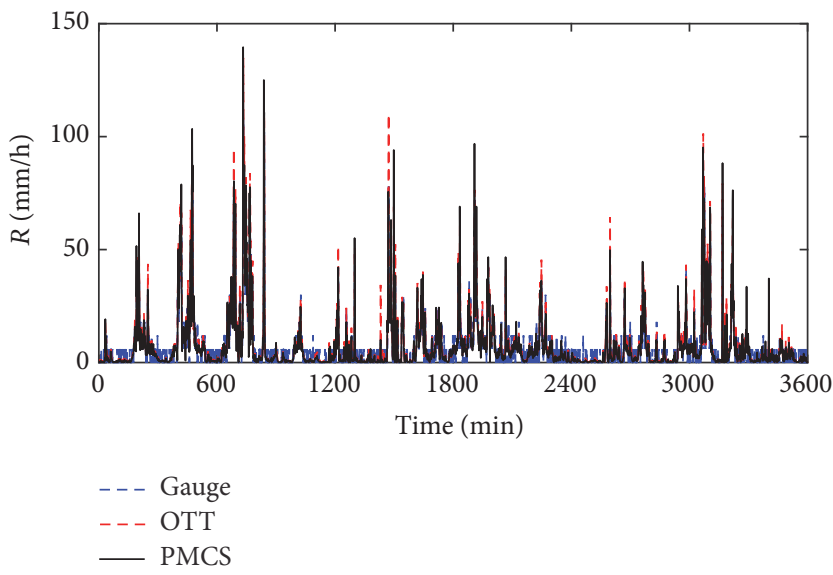

(a)

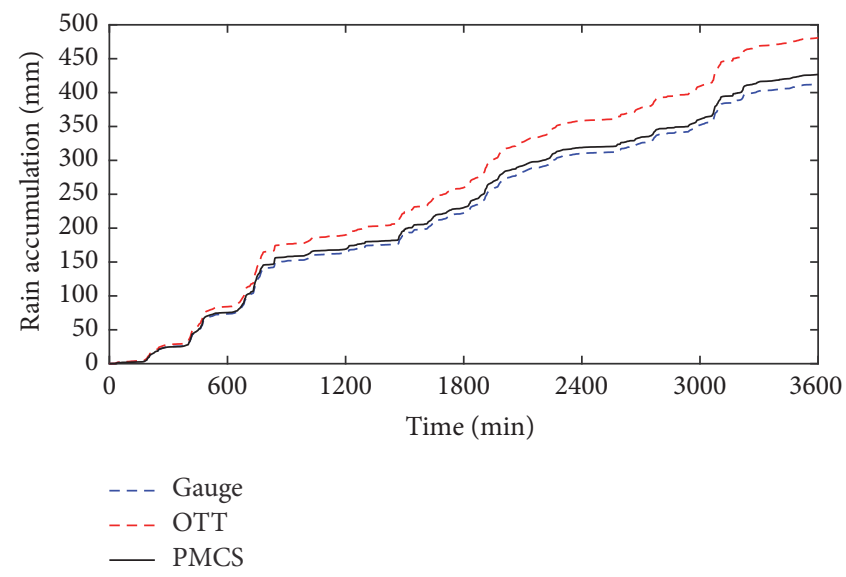

(b)

FIGURE 6: Rain-rate and rainfall accumulations obtained by three instruments.

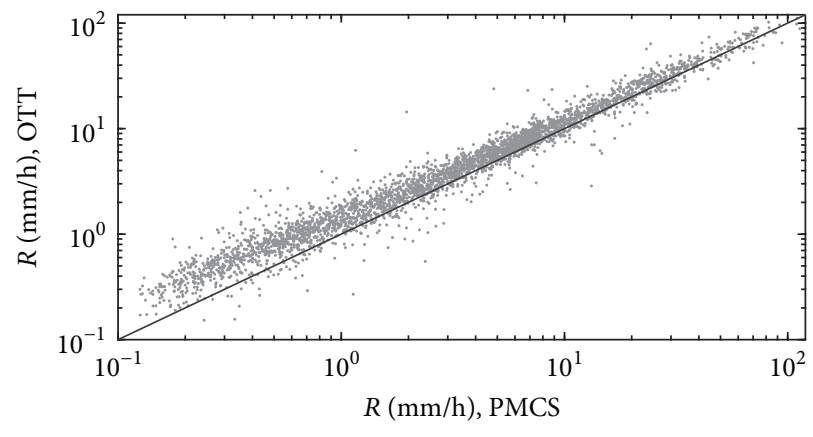

(a)

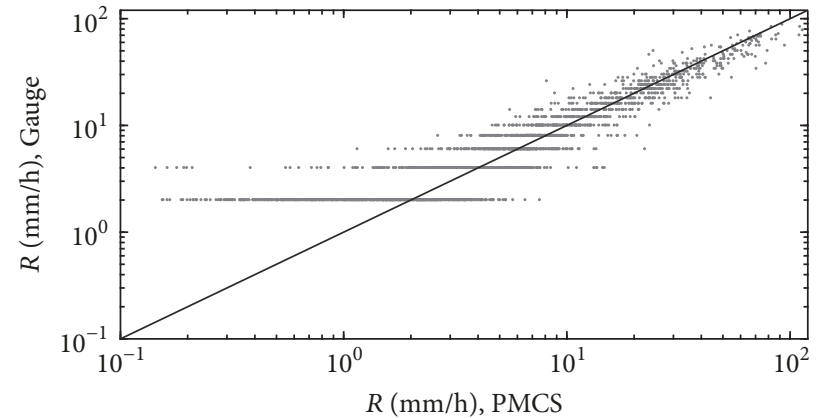

(b)

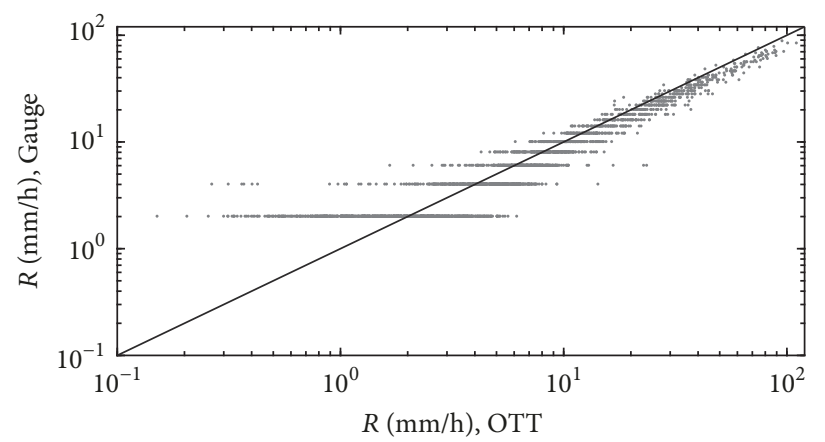

(c)

FIGURE 7: Comparisons of 3 min average rain-rate between three instruments.

TABLE 5: Discrepancies of rain-rate between three instruments in different rain-rate categories.

\begin{tabular}{|c|c|c|c|c|c|c|c|c|c|}
\hline \multirow{2}{*}{$\begin{array}{l}\text { Rain-rate category } \\
(\mathrm{mm} / \mathrm{h})\end{array}$} & \multicolumn{3}{|c|}{ OTT-PMCS } & \multicolumn{3}{|c|}{ PMCS-Gauge } & \multicolumn{3}{|c|}{ OTT-Gauge } \\
\hline & $r^{2}$ & Bias & ab_bias & $r^{2}$ & Bias & ab_bias & $r^{2}$ & Bias & ab_bias \\
\hline $0<R<5$ & 0.90 & $18.3 \%$ & $26.3 \%$ & 0.69 & $4.5 \%$ & $54.3 \%$ & 0.76 & $22.8 \%$ & $50.5 \%$ \\
\hline $5 \leq R<10$ & 0.74 & $14.9 \%$ & $19.6 \%$ & 0.30 & $-4.4 \%$ & $24.1 \%$ & 0.37 & $10.5 \%$ & $21.3 \%$ \\
\hline $10 \leq R<20$ & 0.89 & $10.2 \%$ & $16.0 \%$ & 0.67 & $-0.2 \%$ & $22.5 \%$ & 0.82 & $10.0 \%$ & $16.1 \%$ \\
\hline$R \geq 20$ & 0.93 & $9.4 \%$ & $15.3 \%$ & 0.89 & $6.9 \%$ & $17.8 \%$ & 0.96 & $16.2 \%$ & $17.4 \%$ \\
\hline All & 0.98 & $11.7 \%$ & $17.7 \%$ & 0.96 & $3.3 \%$ & $25.0 \%$ & 0.99 & $15.0 \%$ & $22.7 \%$ \\
\hline
\end{tabular}




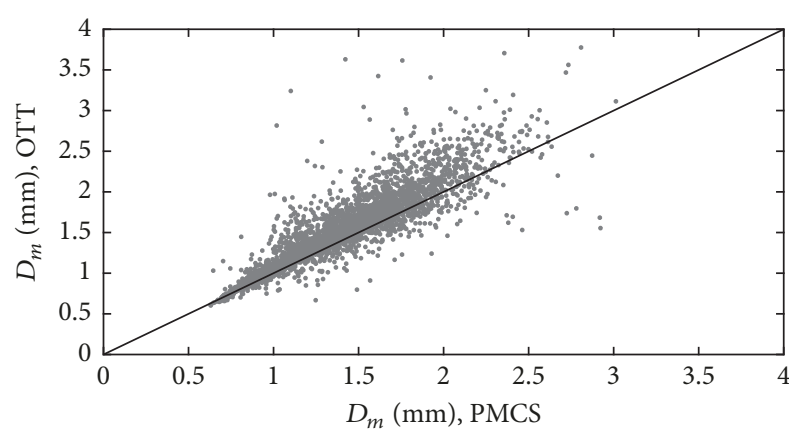

(a)

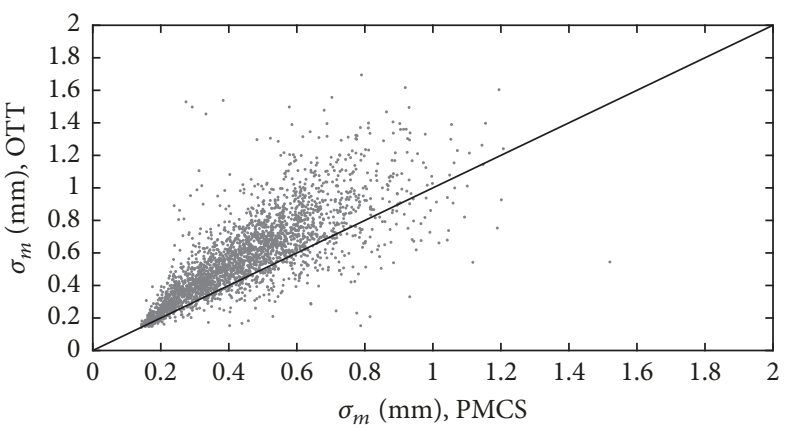

(b)

Figure 8: $D_{m}$ and $\sigma_{m}$ measured by PMCS and OTT.

four categories according to the rain-rate; the average DSDs are shown in Figure 9. For $0.1<R<5 \mathrm{~mm} / \mathrm{h}$, PMCS and OTT measured basically the same DSD with diameter ranging from $0.8 \mathrm{~mm}$ to $3.0 \mathrm{~mm}$; the number density of raindrops with $D<0.8 \mathrm{~mm}$ and $D>3.0 \mathrm{~mm}$ measured by OTT is higher than that measured by PMCS; for $5<R<10 \mathrm{~mm} / \mathrm{h}$, PMCS and OTT can obtain basically the same DSD with diameter smaller than $3.0 \mathrm{~mm}$, and the number density of raindrops with $D>3.0 \mathrm{~mm}$ measured by OTT is higher than that measured by PMCS; for $10 \leq R<20 \mathrm{~mm} / \mathrm{h}$, PMCS can measure more raindrops with $D<0.5 \mathrm{~mm}$ and less raindrops with $D>3.0 \mathrm{~mm}$ than OTT; for $R>20 \mathrm{~mm} / \mathrm{h}$, PMCS and OTT can measure basically the same DSD with diameter ranging from $1.5 \mathrm{~mm}$ to $3.0 \mathrm{~mm}$, and PMCS can measure more raindrops with $D<1.5 \mathrm{~mm}$ and obviously less raindrops with $D>3.0 \mathrm{~mm}$ than OTT.

To summarize the above, the DSDs obtained by PMCS and OTT have a good agreement, compared with OTT; PMCS can measure more small/median raindrops and less large raindrops $(D>3 \mathrm{~mm})$ with the increase of rain-rate; the reason can be attributed to the difference between two sampling principles. The imaging system can record the raindrops' images clearly, while the attenuation of small raindrops on laser transmission is not obvious, causing the underestimation of small raindrops by OTT. On the other hand, large raindrop takes on a smoothed conical shape [23], and its horizontal size is larger than its vertical size; OTT can only measure the horizontal size of raindrops [5], although nonspherical corrections for this exist and overestimation of large raindrops remains. And the capture probability with diameter larger than $3 \mathrm{~mm}$ of PMCS is less than 0.3, causing the underestimation of large raindrops by PMCS.

Based on the DSD measurements, the moment parameters and their deviations are calculated by (12) and (15); the results are shown in Table $6 . r^{2}$ denotes the correlation coefficient, bias denotes the relative deviation, and ab_bias denotes the absolute deviation. For all samples, the correlation coefficients of M2, M3, and M4 between PMCS and OTT are greater than 0.9 , and the relative deviation increases with the increase of moment order; M1 has the minimum relative deviation $0.9 \%$, and the absolute deviations of $\mathrm{M} 1, \mathrm{M} 2$, and $\mathrm{M} 3$ between PMCS and OTT are relatively small, less than $30 \%$. The moment parameters in different rain-rate ranges have the similar features, except that M0 with $5 \leq R<10 \mathrm{~mm} / \mathrm{h}$ has the minimum relative deviation $2.9 \%$, and M2 with $0.1 \leq R<5 \mathrm{~mm} / \mathrm{h}, 10 \leq R<20 \mathrm{~mm} / \mathrm{h}$, and $R \geq 20 \mathrm{~mm} / \mathrm{h}$ has the minimum relative deviations $19.8 \%, 2.1 \%$, and $-5.8 \%$; and $\mathrm{M} 1$ and M2 have the minimum absolute deviation in different rain-rate ranges; the variation of relative deviation and absolute deviation of moments with rain-rate takes on a u-shaped distribution; the relative deviation and absolute deviation of $0.1 \leq R<5 \mathrm{~mm} / \mathrm{h}$ and $R \geq 20 \mathrm{~mm} / \mathrm{h}$ are greater than those of $5 \leq R<10 \mathrm{~mm} / \mathrm{h}$ and $10 \leq R<20 \mathrm{~mm} / \mathrm{h}$. In conclusion, M1, M2, M3, and M4 measured by PMCS and OTT have a good agreement, and M6 has the greatest deviation. The minimum deviation occurs in the rain-rate range $5 \leq R<$ $20 \mathrm{~mm} / \mathrm{h}$ and $10 \leq R<20 \mathrm{~mm} / \mathrm{h}$; the possible reason is that small and large raindrops contribute most to M0 and M6, while the OTT can measure smaller raindrops and larger raindrops than PMCS, and the discrepancy becomes significant with the increase of rain-rate.

Considering that M2, M3, and M4 from PMCS and OTT have better correlation, the M234 order moment method is used to calculate $N_{0}, \mu$, and $\Lambda$ of Gamma distribution, the probability distributions of $N_{0}, \mu$, and $\Lambda$ are shown in Figure 10, and the corresponding peak values, median values, and their probability are listed in Table 7 . It can be found that both peak values and median values obtained by PMCS are greater than those obtained by OTT, and the corresponding probabilities obtained by PMCS are lower than those obtained by OTT; the probabilities of $\log _{10}\left(N_{0}\right)$ and $\Lambda$ have the similar distribution, the probability of PMCS is less than that of OTT when $\log _{10}\left(N_{0}\right)$ and $\Lambda$ are less than their peak values of PMCS, and the probability of PMCS is greater than that of OTT when $\log _{10}\left(N_{0}\right)$ and $\Lambda$ are greater than their peak values of PMCS. Comparing the probability of $\mu$ from OTT with PMCS gives a difference of 0.05 , and the difference between the peak value and median value of $\mu$ from PMCS is 4 , whereas the difference between the peak value and median value of $\mu$ from OTT is only 2 ; when $\mu$ gets greater than its peak value, the probability of PMCS declines more slowly than that of OTT. It can be concluded that there is a good 


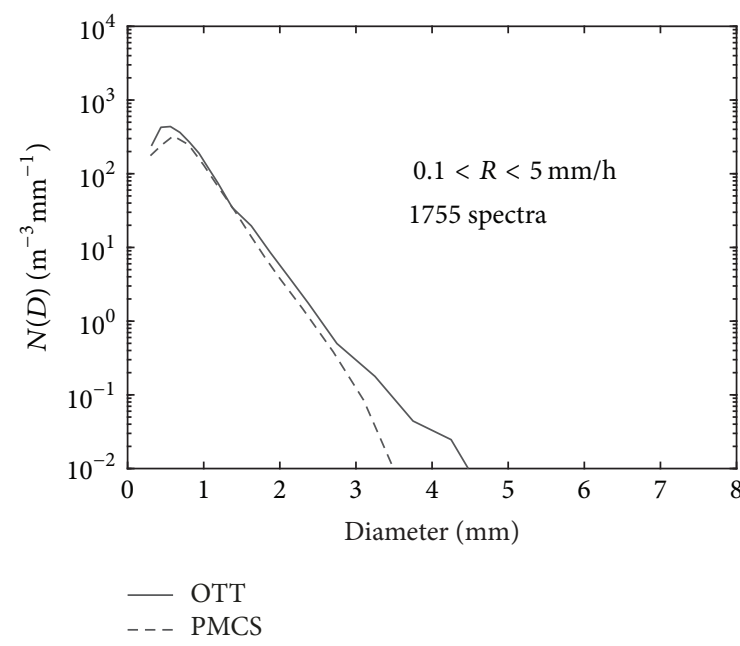

(a)

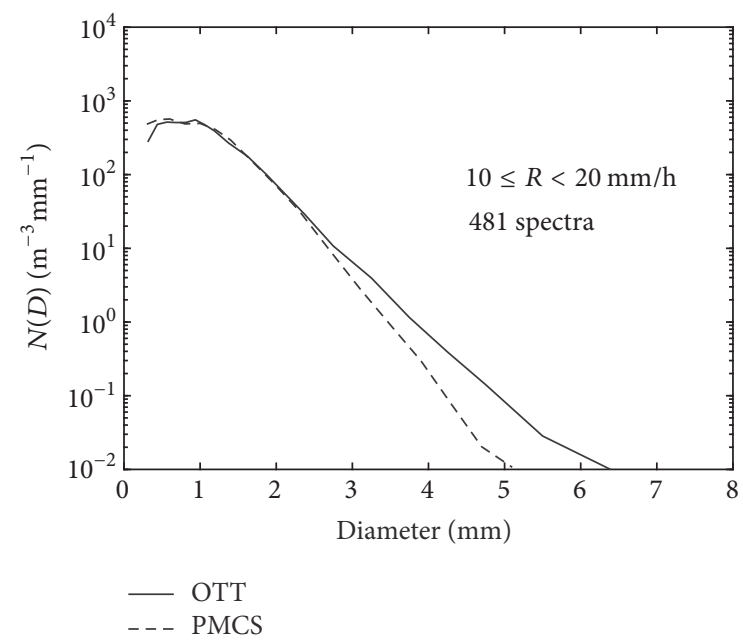

(c)

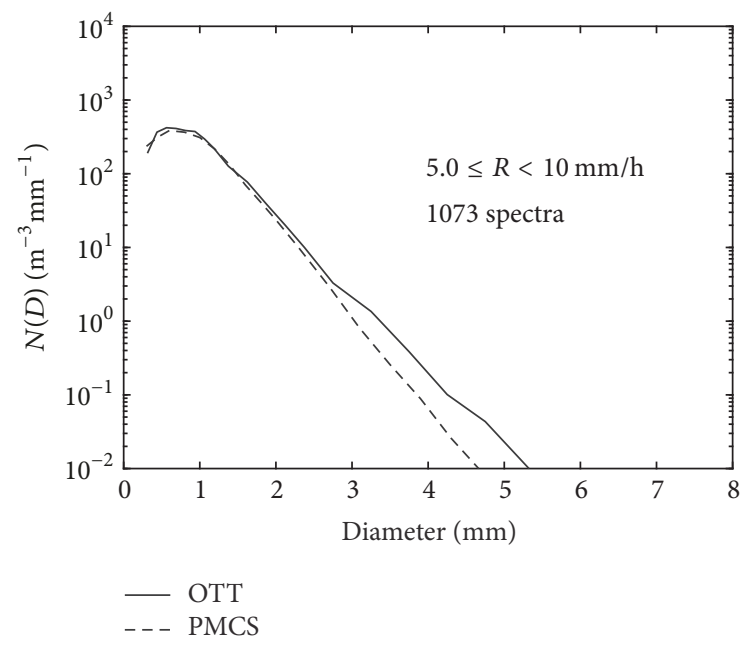

(b)

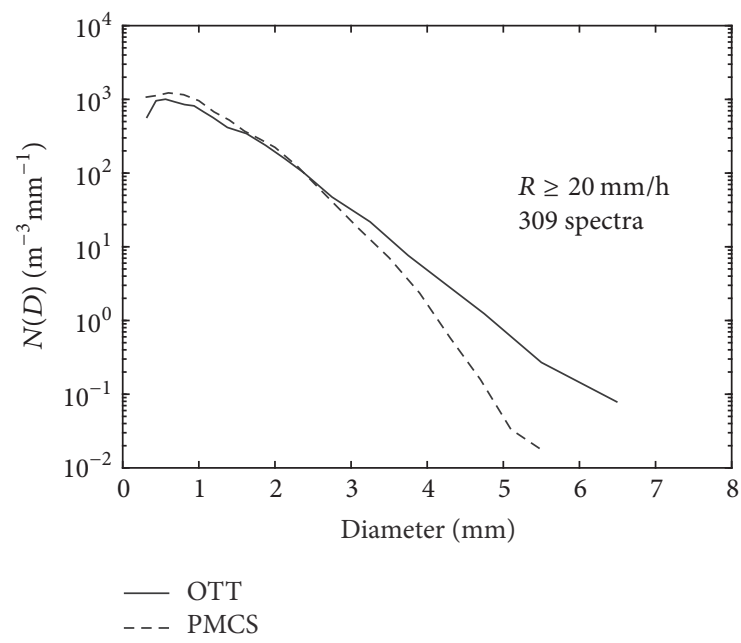

(d)

FIgURE 9: Average DSDs measured by PMCS and OTT in four rain-rate categories.

TABLE 6: Deviation of moment parameters between PMCS and OTT in different rain-rate.

\begin{tabular}{lcccccccc}
\hline Rain-rate $[\mathrm{mm} / \mathrm{h}]$ & PMCS-OTT & M0 & M1 & M2 & M3 & M4 & M5 & M6 \\
\hline \multirow{3}{*}{$0.1 \leq R<5$} & $r^{2}$ & 0.63 & 0.67 & 0.76 & 0.78 & 0.73 & 0.63 & 0.52 \\
& Bias & $22.0 \%$ & $20.5 \%$ & $19.8 \%$ & $22.2 \%$ & $29.5 \%$ & $42.7 \%$ & $62.3 \%$ \\
& ab_bias & $37.8 \%$ & $34.1 \%$ & $32.9 \%$ & $35.8 \%$ & $43.9 \%$ & $57.8 \%$ & $78.2 \%$ \\
\hline \multirow{3}{*}{$5 \leq R<10$} & $r^{2}$ & 0.55 & 0.68 & 0.76 & 0.75 & 0.69 & 0.62 & 0.57 \\
& Bias & $2.9 \%$ & $6.0 \%$ & $8.9 \%$ & $13.7 \%$ & $21.8 \%$ & $33.6 \%$ & $49.1 \%$ \\
& ab_bias & $26.7 \%$ & $22.5 \%$ & $22.6 \%$ & $27.1 \%$ & $36.0 \%$ & $49.2 \%$ & $66.8 \%$ \\
\hline \multirow{3}{*}{$10 \leq R<20$} & $r^{2}$ & 0.34 & 0.54 & 0.69 & 0.76 & 0.74 & 0.71 & 0.67 \\
& Bias & $-10.1 \%$ & $-2.9 \%$ & $2.1 \%$ & $8.2 \%$ & $17.5 \%$ & $31.1 \%$ & $49.5 \%$ \\
& ab_bias & $34.0 \%$ & $24.0 \%$ & $20.7 \%$ & $22.4 \%$ & $29.4 \%$ & $41.5 \%$ & $59.0 \%$ \\
\hline \multirow{3}{*}{$R \geq 20$} & $r^{2}$ & 0.43 & 0.54 & 0.67 & 0.76 & 0.78 & 0.75 & 0.71 \\
& Bias & $-25.8 \%$ & $-15.9 \%$ & $-5.8 \%$ & $6.7 \%$ & $22.5 \%$ & $41.9 \%$ & $64.3 \%$ \\
\hline \multirow{2}{*}{ All } & ab_bias & $48.1 \%$ & $34.1 \%$ & $25.1 \%$ & $23.2 \%$ & $31.4 \%$ & $47.0 \%$ & $67.8 \%$ \\
\hline & $r^{2}$ & 0.66 & 0.81 & 0.90 & 0.92 & 0.91 & 0.88 & 0.82 \\
& Bias & $-1.7 \%$ & $0.9 \%$ & $4.0 \%$ & $10.5 \%$ & $21.8 \%$ & $38.2 \%$ & $59.1 \%$ \\
& ab_bias & $36.7 \%$ & $28.8 \%$ & $24.7 \%$ & $25.4 \%$ & $32.9 \%$ & $46.8 \%$ & $66.4 \%$ \\
\hline
\end{tabular}


TABLE 7: Peak values, median values, and corresponding probability of probability distributions of $N_{0}, \mu$, and $\Lambda$ of Gamma function.

\begin{tabular}{lccccccc}
\hline \multirow{2}{*}{ Gamma parameter } & \multicolumn{2}{c}{ Peak value } & \multicolumn{2}{c}{ Probability of peak value } & \multicolumn{2}{c}{ Median value } & \multicolumn{2}{c}{$\begin{array}{c}\text { Probability of } \\
\text { median value }\end{array}$} \\
& PMCS & OTT & PMCS & OTT & PMCS & OTT & PMCS \\
\hline $\log _{10}\left(N_{0}\right)$ & 4.8 & 4.3 & 0.11 & 0.15 & 6.3 & 5.3 & 0.06 \\
$\mu$ & 4.5 & 3.5 & 0.08 & 0.13 & 8.5 & 5.5 & 0.06 \\
$\Lambda$ & 5.5 & 3.5 & 0.10 & 0.13 & 8.5 & 6.5 & 0.05 \\
\hline
\end{tabular}
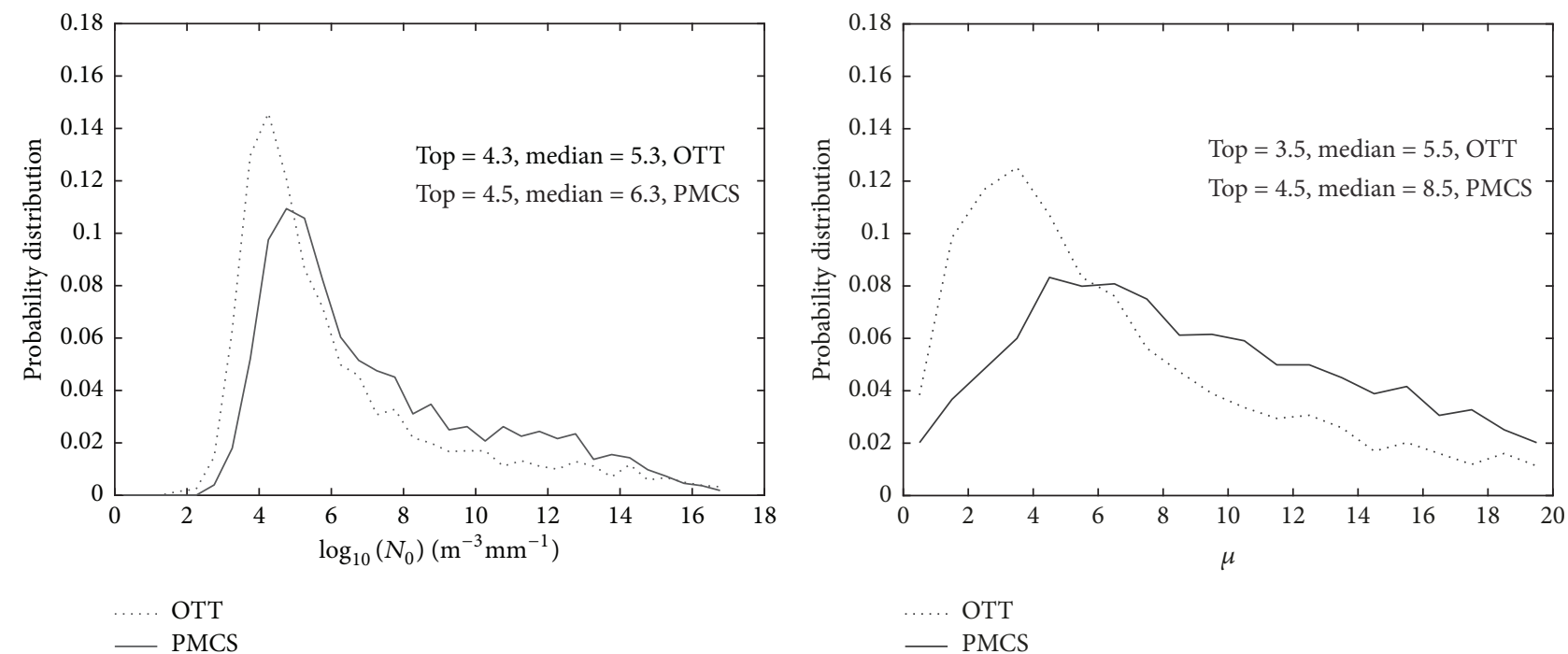

(a)

(b)

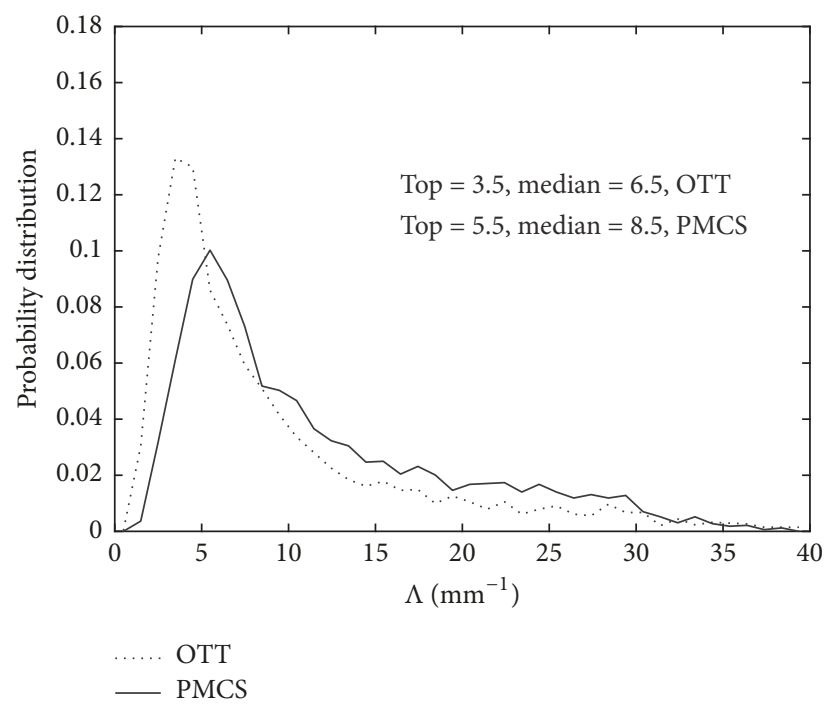

(c)

Figure 10: Probability distribution of $N_{0}, \mu, \Lambda$ of Gamma function.

agreement between the Gamma parameters of PMCS and OTT, while the PMCS can obtain a more complex DSD variation because of the capture probability of PMCS.

$\mu-\Lambda$ and $Z-R$ relationship obtained by PMCS and OTT are fitted by least square method and shown in Figure 11; the corresponding expressions are listed in Table 8. $\mu$ and $\Lambda$ measured by both PMCS and OTT follow a typical quadratic function relationship, the fitted correlation coefficients are 0.911 and 0.914 , and the PMCS has higher $\mu$ than OTT, causing the $\mu-\Lambda$ expression obtained by PMCS to have a greater quadratic coefficient and a smaller monomial coefficient than those obtained by OTT. $Z$ and $R$ measured by both PMCS and OTT follow an exponential function relationship, and PMCS has a more concentrated $Z-R$ scatter than OTT; the 


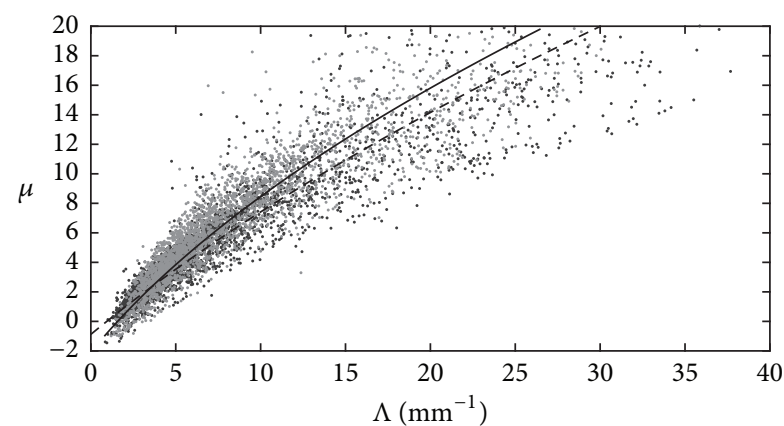

Raw OTT Raw PMCS
- - - OTT fitness _ PMCS fitness

(a)

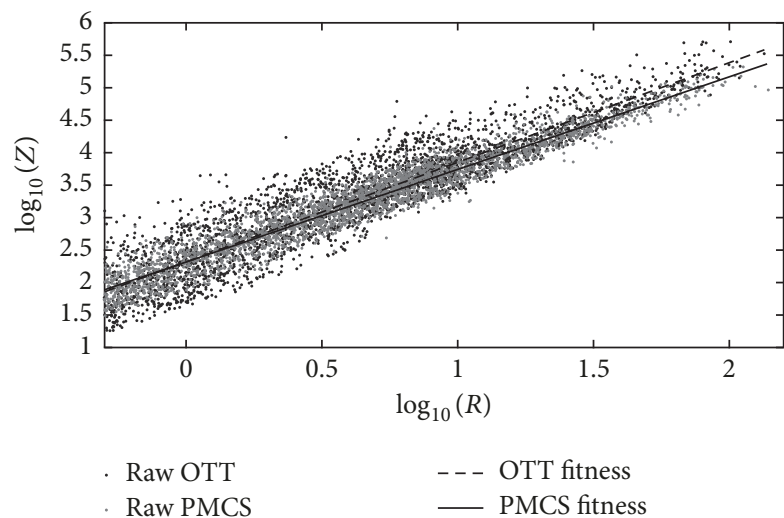

(b)

FIGURE 11: $\mu-\Lambda$ and $Z-R$ relationship obtained by PMCS and OTT.

TABLE 8: Expression of $\mu-\Lambda$ and $Z-R$ relationship obtained by PMCS and OTT.

\begin{tabular}{lcc}
\hline Instrument & Fitted equation & $r^{2}$ \\
\hline PMCS & $\Lambda=0.023 \mu^{2}+0.803 \mu+1.576$ & 0.911 \\
PMCS & $Z=231.7 R^{1.426}$ & 0.969 \\
OTT & $\Lambda=0.019 \mu^{2}+1.085 \mu+0.939$ & 0.914 \\
OTT & $Z=231.8 R^{1.534}$ & 0.931 \\
\hline
\end{tabular}

coefficient of fitted $Z-R$ relationship measured by PMCS is very close to that measured by OTT, only the fitted curve of PMCS is slightly lower than that of OTT, and the correlation coefficients are 0.969 and 0.931 separately.

\section{Conclusions}

Sampling parameters of different disdrometers have various impacts on the rainfall properties estimation. Aiming at the self-developed precipitation microphysical characteristics sensor (PMCS), the sampling process of raindrops by PMCS based on a particle-by-particle Monte-Carlo model was simulated, the sampling effect of different bin sizes on DSD measurement of PMCS was discussed, and the optimum sampling bin sizes were proposed.

The simulation results of five sampling schemes of bin sizes in four rain-rate categories (light rainfall, moderate rainfall, heavy rainfall, and rainstorm) show that the raw capture DSD has a significant fluctuation variation influenced by the capture probability, and a certain number of large raindrops are missed; compared with the raw capture DSD, there are good consistencies between the inversed DSDs from 4 interval schemes and real DSD; the reason is that the appropriate sampling bin size and width can reduce the impact of variation of raindrop number on DSD shape. The scheme interval 4 has the minimum relative error and absolute error on the whole; therefore, the scheme interval 4 is adopted as the optimal sampling bin sizes of PMCS.

The field measurement of a PMCS, an OTT PARSIVEL disdrometer, and a tipping bucket rain Gauge shows that the rain-rate and rainfall accumulations have good consistencies between PMCS, OTT, and Gauge; the DSDs obtained by PMCS and OTT have a good agreement, compared with OTT, and PMCS can measure more small/median raindrops and less large raindrops $(D>3 \mathrm{~mm})$ with the increase of rainrate; the probability of $N_{0}, \mu$, and $\Lambda$ shows that there is a good agreement between the Gamma parameters of PMCS and OTT, while the PMCS can obtain a more complex DSD variation because of the capture probability of PMCS; the fitted $\mu-\Lambda$ and $Z-R$ relationship measured by PMCS is close to that measured by OTT, which validates the performance of PMCS on rain-rate, rainfall accumulation, and DSD related parameters.

\section{Conflicts of Interest}

The authors declare that they have no conflicts of interest.

\section{Authors' Contributions}

All authors were involved in designing and discussing the study. This research idea was conceived of by Xichuan Liu and Taichang Gao. The experiments were designed and performed by Xichuan Liu and Xiaojian Shu. The data were analyzed and interpreted by Xichuan Liu and Yuntao Hu. The manuscript was written by Xichuan Liu and Xiaojian Shu.

\section{Acknowledgments}

This work is supported by the National Natural Science Foundation of China (Grant nos. 41327003, 41505135, and 41475020) and the Natural Science Foundation of Jiangsu Province (Grant no. BK20150708). The PMCS was developed with support from Ying EnTe Environment Technique Co., Ltd., Nanjing, China.

\section{References}

[1] S. Michaelides, "Precipitation: Advances in measurement, estimation and prediction," Precipitation: Advances in Measurement, Estimation and Prediction, pp. 1-540, 2008. 
[2] G. Kathiravelu, T. Lucke, and P. Nichols, "Rain drop measurement techniques: a review," Water (Switzerland), vol. 8, no. 1, article 29, 2016.

[3] N. J. Kinar and J. W. Pomeroy, "Measurement of the physical properties of the snowpack," Reviews of Geophysics, vol. 53, no. 2, pp. 481-544, 2015.

[4] M. Fernández-Raga, C. Palencia, C. Tomás, A. I. Calvo, A. Castro, and R. Fraile, "Rain research with disdrometers: a bibliometric review," Atmospheric Measurement Techniques Discussions, vol. 4, no. 5, pp. 6041-6068, 2011.

[5] K. Friedrich, S. Higgins, F. J. Masters, and C. R. Lopez, "Articulating and stationary PARSIVEL disdrometer measurements in conditions with strong winds and heavy rainfall," Journal of Atmospheric and Oceanic Technology, vol. 30, no. 9, pp. 20632080, 2013.

[6] W. L. Randeu, T. Kozu, T. Shimomai, H. Hashiguchi, and M. Schönhuber, "Raindrop axis ratios, fall velocities and size distribution over Sumatra from 2D-Video disdrometer measurement," Atmospheric Research, vol. 119, pp. 23-37, 2013.

[7] B. M. Notaroš, V. N. Bringi, C. Kleinkort et al., "Accurate characterization of winter precipitation using multi-angle snowflake camera, visual hull, advanced scattering methods and polarimetric radar," Atmosphere, vol. 7, no. 6, article no. 81, 2016.

[8] A. Tokay, A. Kruger, and W. F. Krajewski, "Comparison of drop size distribution measurements by impact and optical disdrometers," Journal of Applied Meteorology and Climatology, vol. 40, no. 11, pp. 2083-2097, 2001.

[9] L. C. Sieck, S. J. Burges, and M. Steiner, "Erratum: Challenges in obtaining reliable measurements of point rainfall (Water Resources Research (2007) 43, (W01420) DOI: 10.1029/2005WR004519)," Water Resources Research, vol. 43, no. 6, Article ID W06701, 2007.

[10] M. Thurai, W. A. Petersen, A. Tokay, C. Schultz, and P. Gatlin, "Drop size distribution comparisons between Parsivel and 2-D video disdrometers," Advances in Geosciences, vol. 30, pp. 3-9, 2011.

[11] A. Tokay, W. A. Petersen, P. Gatlin, and M. Wingo, "Comparison of raindrop size distribution measurements by collocated disdrometers," Journal of Atmospheric and Oceanic Technology, vol. 30, no. 8, pp. 1672-1690, 2013.

[12] X. C. Liu, T. C. Gao, and L. Liu, "A comparison of rainfall measurements from multiple instruments," Atmospheric Measurement Techniques, vol. 6, no. 7, pp. 1585-1595, 2013.

[13] T. Sarkar, S. Das, and A. Maitra, "Assessment of different raindrop size measuring techniques: Inter-comparison of Doppler radar, impact and optical disdrometer," Atmospheric Research, vol. 160, pp. 15-27, 2015.

[14] R. Uijlenhoet, J. M. Porrà, D. S. Torres, and J.-D. Creutin, “Analytical solutions to sampling effects in drop size distribution measurements during stationary rainfall: Estimation of bulk rainfall variables," Journal of Hydrology, vol. 328, no. 1-2, pp. 6582, 2006.

[15] A. Berne and R. Uijlenhoet, "A simulation approach to sampling effects in raindrop size distribution measurements in non-stationary rain," in Proceedings of the 11th Conference on Mesoscale Processes and the 32nd Conference on Radar Meteorology, pp. 377-381, usa, October 2005.

[16] J. Jaffrain and A. Berne, "Experimental quantification of the sampling uncertainty associated with measurements from Parsivel disdrometers," Journal of Hydrometeorology, vol. 12, no. 3, pp. 352-370, 2011.
[17] M. Marzuki, W. L. Randeu, M. Schönhuber, V. N. Bringi, T. Kozu, and T. Shimomai, "Raindrop size distribution parameters of distrometer data with different bin sizes," IEEE Transactions on Geoscience and Remote Sensing, vol. 48, no. 8, pp. 3075-3080, 2010.

[18] R. Checa-Garcia, A. Tokay, and F. J. Tapiador, "Binning effects on in-situ raindrop size distribution measurements," Atmospheric Measurement Techniques Discussions, vol. 7, no. 3, pp. 2339-2379, 2014.

[19] X. C. Liu, T. C. Gao, and L. Liu, "A video precipitation sensor for imaging and velocimetry of hydrometeors," Atmospheric Measurement Techniques, vol. 7, no. 7, pp. 2037-2046, 2014.

[20] R. Uijlenhoet, D. S. Torres, and J. D. Creutin, "Analytical solutions to sampling fluctuations in raindrop size distribution measurements," in Proceedings of the 30th International Conference on Radar Meteorology, 2001.

[21] J. A. Smith, "Marked point process models of raindrop-size distributions," Journal of Applied Meteorology and Climatology, vol. 32, no. 2, pp. 284-296, 1993.

[22] A. Tokay and D. A. Short, "Evidence from tropical raindrop spectra of the origin of rain from stratiform versus convective clouds," Journal of Applied Meteorology and Climatology, vol. 35, no. 3, pp. 355-371, 1996.

[23] M. Thurai, E. Chobanyan, V. N. Bringi, and B. M. Notaroš, "Large raindrops against melting hail: Calculation of specific differential attenuation, phase and reflectivity," IEEE Electronics Letters, vol. 51, no. 15, pp. 1140-1142, 2015. 

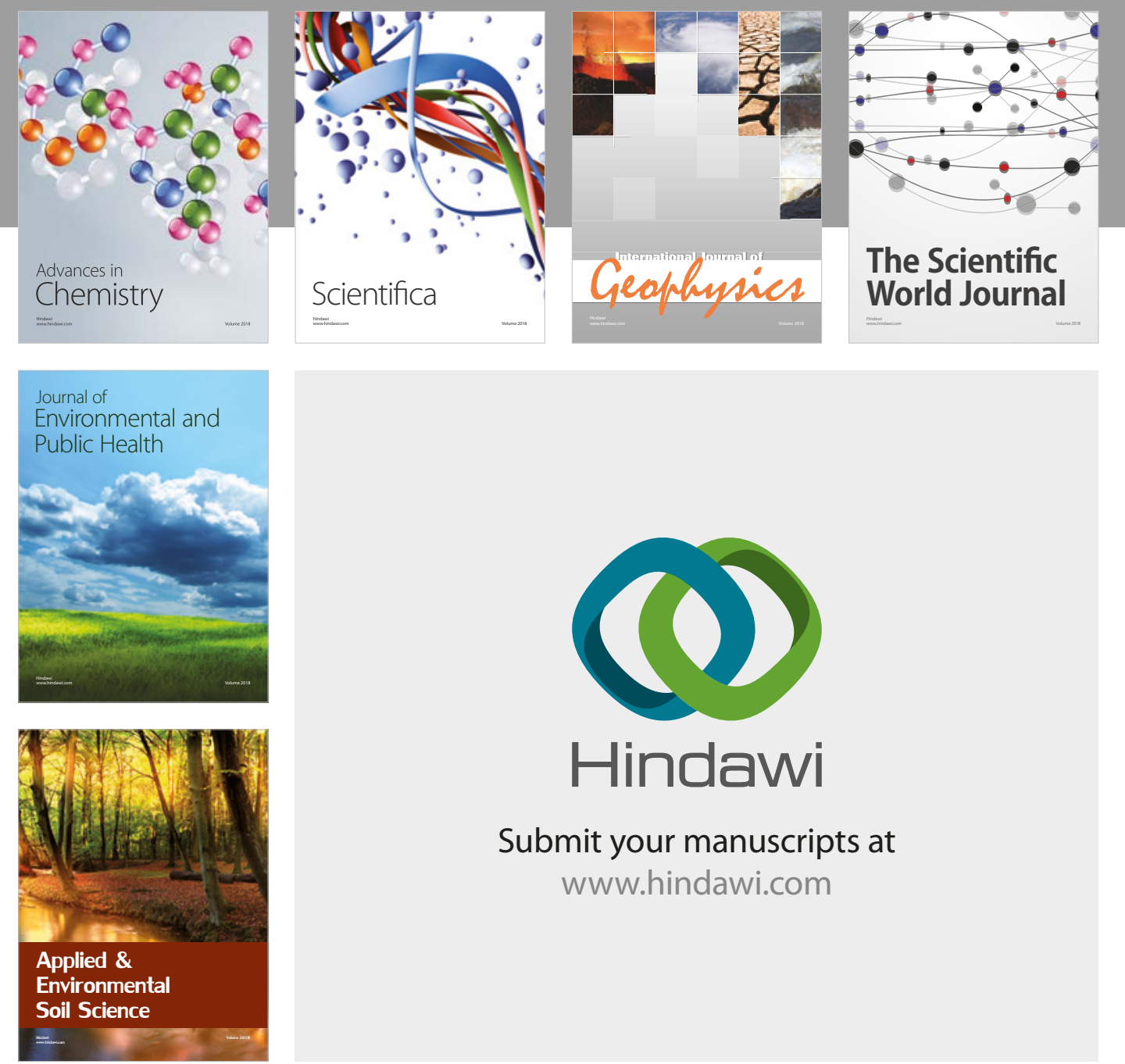

The Scientific

\section{World Journal}
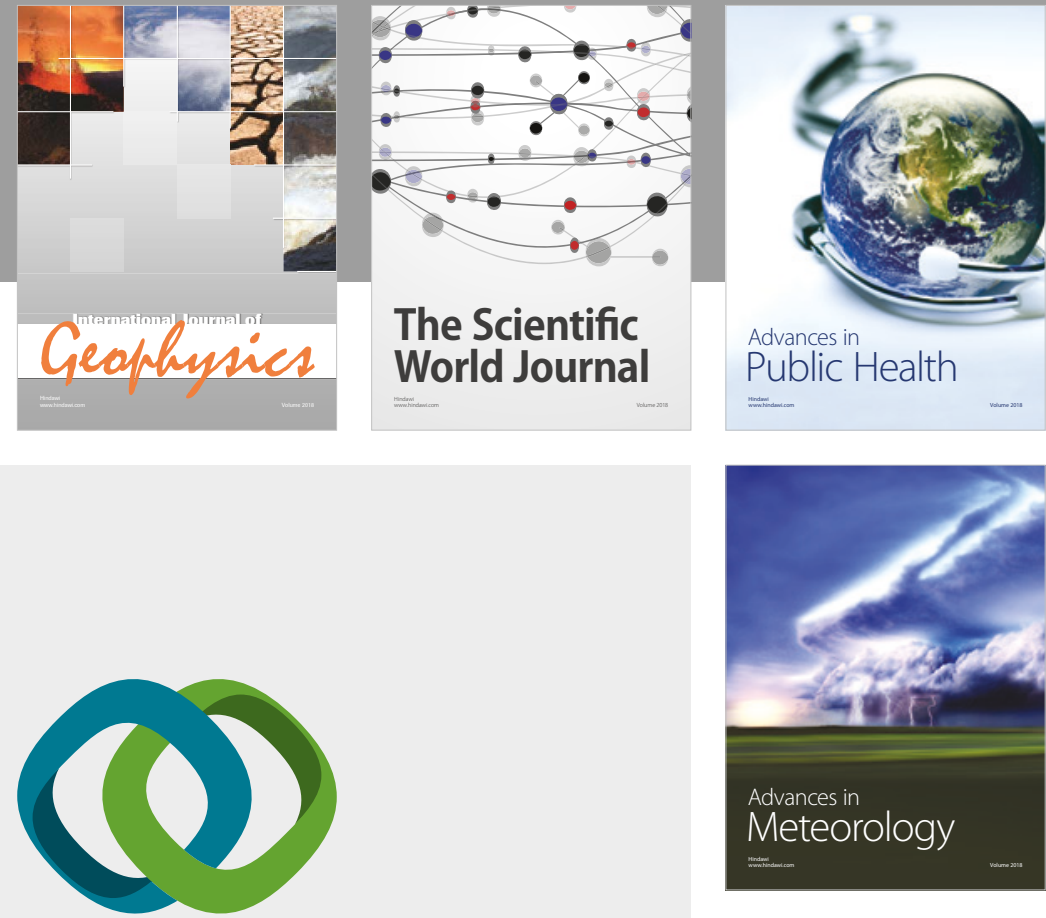

Advan

Public Health

\section{Hindawi}

Submit your manuscripts at

www.hindawi.com
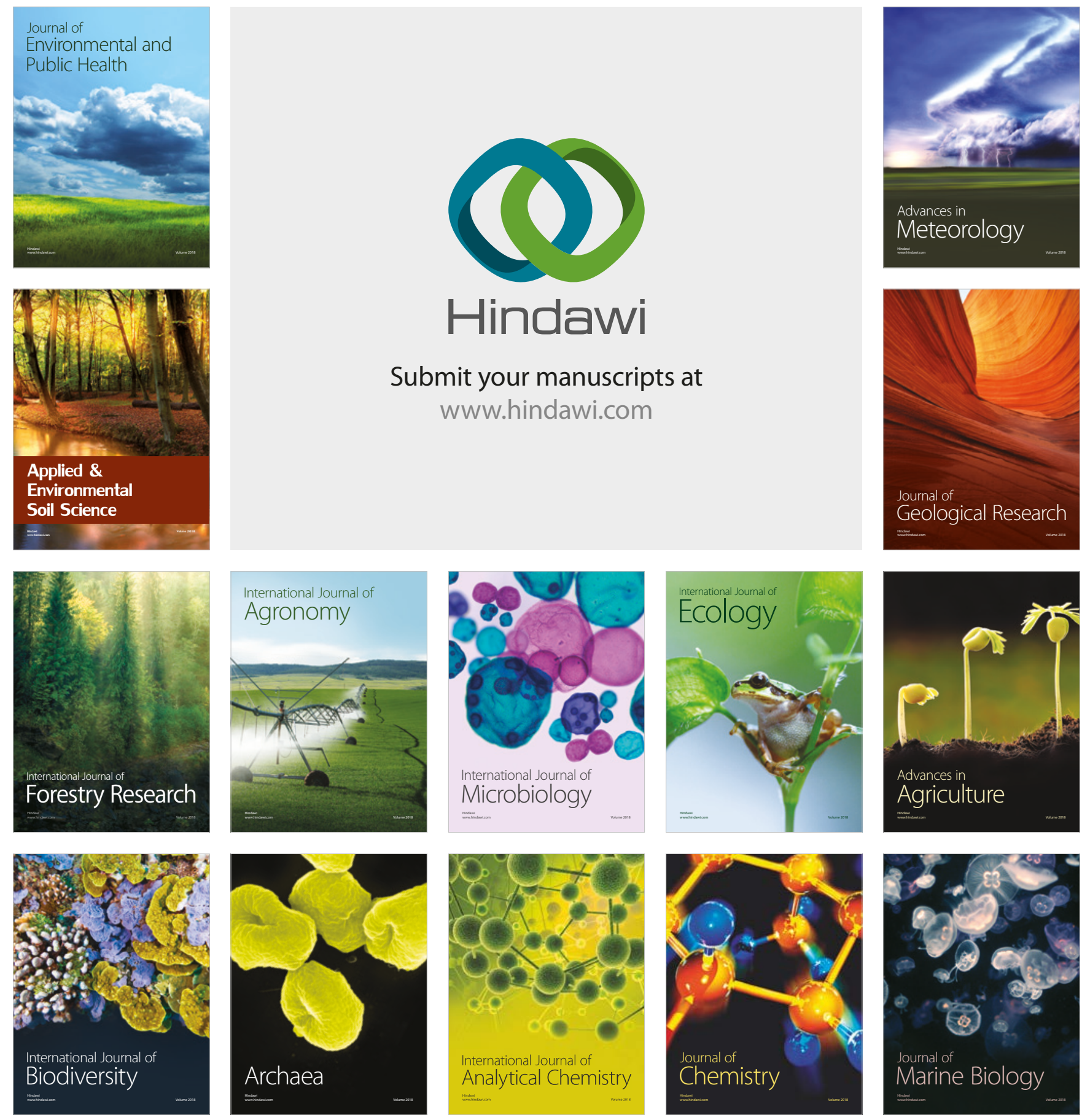